\title{
Targeting nuclear receptor NR4A1-dependent adipocyte progenitor quiescence promotes metabolic adaptation to obesity
}

\author{
Yang Zhang, ${ }^{1,2}$ Alexander J. Federation, ${ }^{3,4}$ Soomin Kim, ${ }^{1}$ John P. O'Keefe, ${ }^{1}$ Mingyue Lun, ${ }^{1}$ Dongxi Xiang, ${ }^{1,2}$ Jonathan D. Brown, ${ }^{2,5,6}$ \\ and Matthew L. Steinhauser ${ }^{1,2,5,7}$ \\ 'Department of Medicine, Division of Genetics, Brigham and Women's Hospital, Boston, Massachusetts, USA. ${ }^{2}$ Harvard Medical School, Boston, Massachusetts, USA. ${ }^{3}$ Department of Medical Oncology, \\ Dana Farber Cancer Institute, Boston, Massachusetts, USA. ${ }^{4}$ Altius Institute for Biomedical Sciences, Seattle, Washington, USA. ${ }^{5}$ Department of Medicine, Division of Cardiovascular Medicine, Brigham and \\ Women's Hospital, Boston, Massachusetts, USA. ${ }^{6}$ Department of Medicine, Division of Cardiovascular Medicine, Vanderbilt University Medical Center, Nashville, Tennessee, USA. ${ }^{7}$ Broad Institute of MIT and \\ Harvard, Cambridge, Massachusetts, USA.
}

\begin{abstract}
Adipocyte turnover in adulthood is low, suggesting that the cellular source of new adipocytes, the adipocyte progenitor (AP), resides in a state of relative quiescence. Yet the core transcriptional regulatory circuitry (CRC) responsible for establishing a quiescent state and the physiological significance of AP quiescence are incompletely understood. Here, we integrate transcriptomic data with maps of accessible chromatin in primary APs, implicating the orphan nuclear receptor NR4A1 in AP cell-state regulation. NR4A1 gain and loss of function in APs ex vivo decreased and enhanced adipogenesis, respectively. Adipose tissue of $\mathrm{Nr}_{4 a 1^{-/-}}$mice demonstrated higher proliferative and adipogenic capacity compared with that of WT mice. Transplantation of $\mathrm{Nr}_{4 \mathrm{ar}}{ }^{-/-}$APs into the subcutaneous adipose tissue of WT obese recipients improved metrics of glucose homeostasis relative to administration of WT APs. Collectively, these data identify NR4A1 as a previously unrecognized constitutive regulator of AP quiescence and suggest that augmentation of adipose tissue plasticity may attenuate negative metabolic sequelae of obesity.
\end{abstract}

\section{Introduction}

The obesity epidemic is a major public health concern due to its close association with cardiometabolic diseases, including T2DM $(1,2)$. Expansion of adipose tissue (AT) - the defining feature of obesity - plays a key role in metabolic disease, as the pathological changes in AT impair systemic glucose and lipid homeostasis. Adipocyte development, which occurs by the proliferation and differentiation of adipocyte progenitors (APs) (adipogenesis), represents one determinant of metabolic health. In the extreme, failure of adipocyte development as occurs in some lipodystrophic diseases causes severe systemic insulin resistance (3). More subtle impairments of adipogenesis due to genetic variants in regulators of adipocyte differentiation, such as peroxisome proliferatoractivated receptor $\gamma(P P A R G)$, are also associated with systemic metabolic disease (4-6). These data highlight the significance of adipocyte development and provide a rationale for dissecting the molecular mechanisms controlling AP cell-fate decisions.

Retrospective carbon birth-dating of adipocytes isolated from subcutaneous AT (SAT) suggests an annual adipocyte birth rate in adult humans of approximately $10 \%$ (7). We have prospectively studied adipogenesis in vivo in mice and humans with stable

Conflict of interest: The authors have declared that no conflict of interest exists. License: Copyright 2018, American Society for Clinical Investigation.

Submitted: October 27, 2017; Accepted: August 23, 2018.

Reference information: / Clin Invest. 2018;128(11):4898-4911.

https://doi.org/10.1172/JCI98353. isotope tracers of cell division and detected similar rates of adipocyte birth in SAT $(8,9)$. Such a low daily birth rate establishes that a small fraction of APs are actively replicating and differentiating. This calculus holds even for visceral AT (VAT), in which turnover is more rapid relative to $\operatorname{SAT}(9,10)$. Yet the molecular mechanisms involved in maintaining this putative state of AP quiescence are incompletely understood, in part because much of the molecular regulation of adipogenesis has been elucidated using in vitro models of differentiation with preadipocyte cell lines, which may not recapitulate all aspects of early adipocyte development. In particular, it remains unclear which transcriptional regulators control proximal fate decisions in APs, providing a rationale for exploring the molecular regulation of AP quiescence. In this study, we leveraged transcriptomic data together with maps of accessible chromatin to develop a model of the core regulatory circuitry in primary APs. We then applied functional genomics approaches to validate a lead candidate, identifying the orphan nuclear receptor NR4A1, also known by the alias NUR77, as a critical node in the regulatory control of AP quiescence and a negative regulator of metabolically beneficial AT plasticity.

\section{Results}

Identification of candidate transcriptional regulators of AP function. AT mass increases dramatically between birth and adulthood (Supplemental Figure 1, A and B; supplemental material available online with this article; https://doi.org/10.1172/JCI98353DS1), which is attributable to some combination of increased adipocyte number (i.e., hyper- 
A

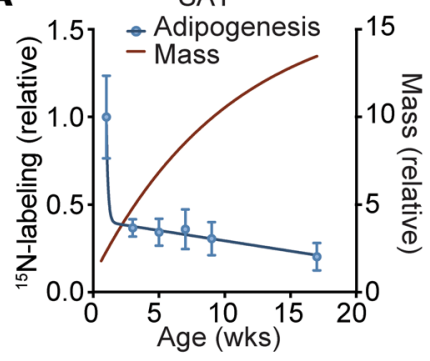

B

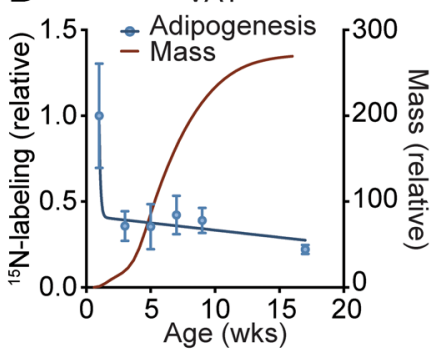

C

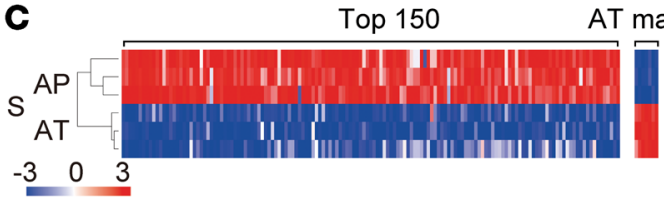

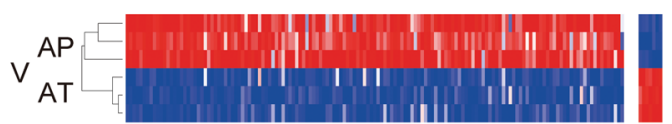

D

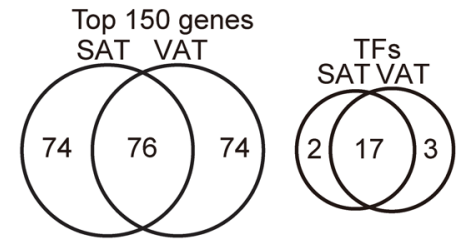

$\mathbf{F}$

E

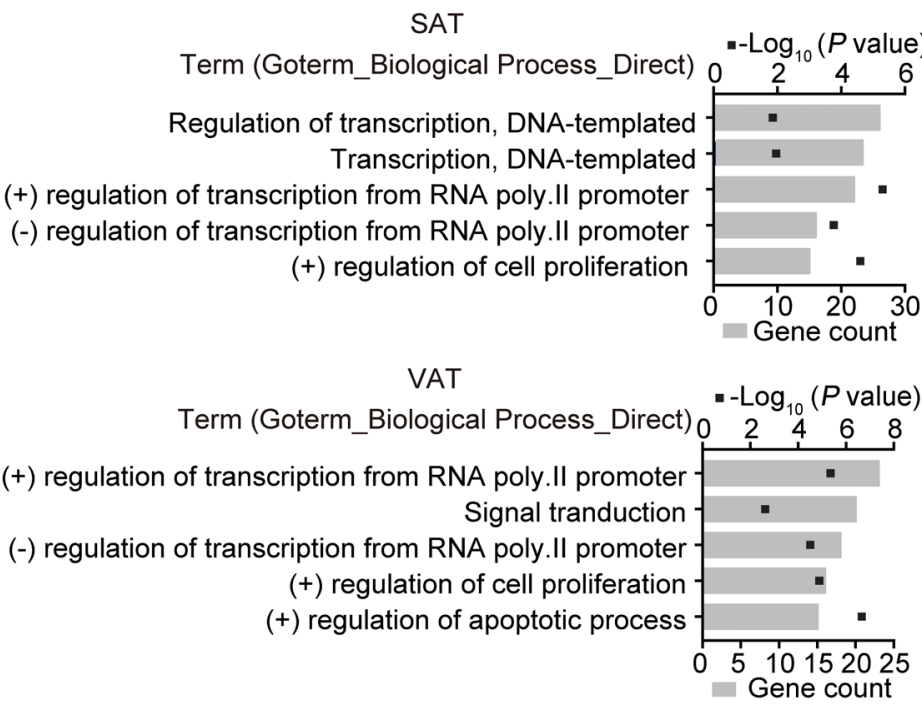

G

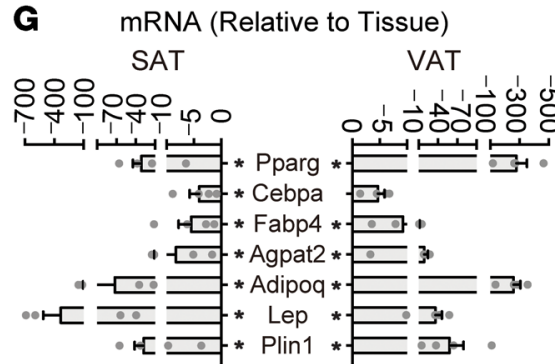

Figure 1. Identification of candidate transcriptional regulators of AP function. (A and B) Relative adipogenesis measured as a function of postnatal age. ${ }^{15} \mathrm{~N}$-thymidine administered for 2 weeks to mice of various ages ranging from less than 24 hours old to 15 weeks old. ${ }^{15} \mathrm{~N}$-label in the adipocyte fraction, indicative of proliferating and differentiating APs, was measured by IRMS. Relative mass provided as reference in red (see also Supplemental Figure 1, A and B). Data expressed as mean \pm SEM. $n=4-11$ mice per time point. (C) Microarray analyses of freshly isolated AP-rich stromal vascular fractions (see also Supplemental Figure $1 C$ ) relative to whole AT from the same mice ( $n=3$ mice). Heatmap representation of top 150 upregulated AP genes and 7 representative adipocyte markers in subcutaneous (S) and visceral (V) depots. (D) Left: Venn diagram of the overlap of the top 150 genes between AT depots. Right: TF fraction of the top 150 genes. (E) Top 5 GO terms for biological processes of differently expressed genes annotated by DAVID. (F) Real-time qPCR of freshly isolated APs, inclusive of the $22 \mathrm{TFs}$ in $\mathbf{C}$ and expressed relative to unfractionated AT. (G) Relative expression of terminal adipocyte markers in APs relative to unfractionated AT. (F and $\mathbf{G})$ Data displayed as bar graphs $\pm \mathrm{SEM}$, with dots showing individual values; $n=4$ mice. ${ }^{*} P<0.05,2$-tailed, unpaired $t$ test (pass Shapiro-Wilk normality test) and Mann-Whitney $U$ test (values are not from Gaussian distribution).

plasia) and enlargement of existing adipocytes (i.e., hypertrophy). To more precisely define the postnatal dynamics of adipocyte hyperplasia, we administered ${ }^{15} \mathrm{~N}$-thymidine for 2-week intervals starting within 24 hours of birth and then quantified label incorporation in the adipocyte fraction, which is the end result of replicating and differentiating APs (9). In SAT and VAT, we measured a marked decline in hyperplasia in mice older than 2 weeks, despite continued increase in AT mass (Figure 1, A and B). These data demonstrate that the postnatal developmental surge in adipogenesis peaks within 2 postnatal weeks and provided the rationale to focus our interrogation of AP quiescence on mice aged beyond this apparent developmental switch from hyperplastic to hypertrophic growth (11).

We sought to rapidly isolate APs from the stromal vascular fraction of AT in which they reside, reflecting our goal to preserve in vivo context. We tested a negative selection strategy, utilizing antibody-coated magnetic beads to deplete hematopoietic cells (lineage ${ }^{+}$) and $\mathrm{CD} 1^{+}$endothelial cells from the stromal vascular fraction (Supplemental Figure 1, C and D) after enzymatic dissoci- 
A

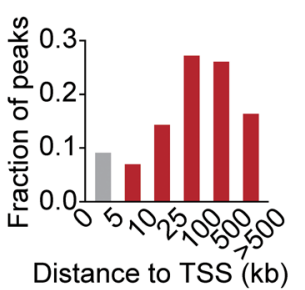

B

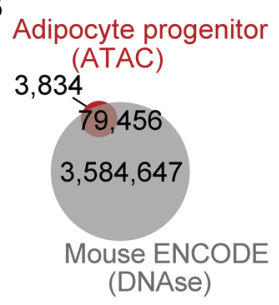

C

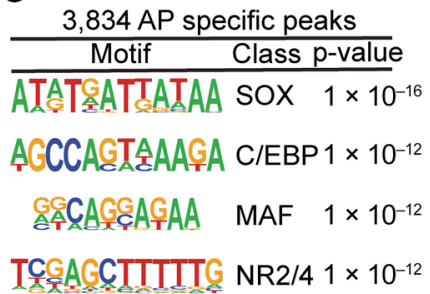

D

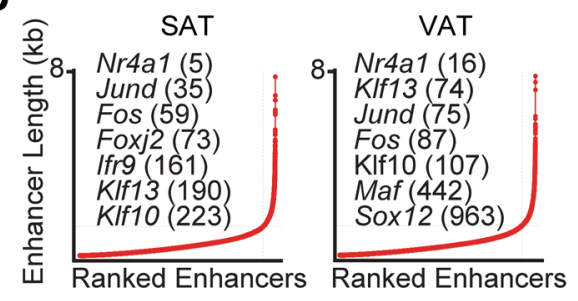

E

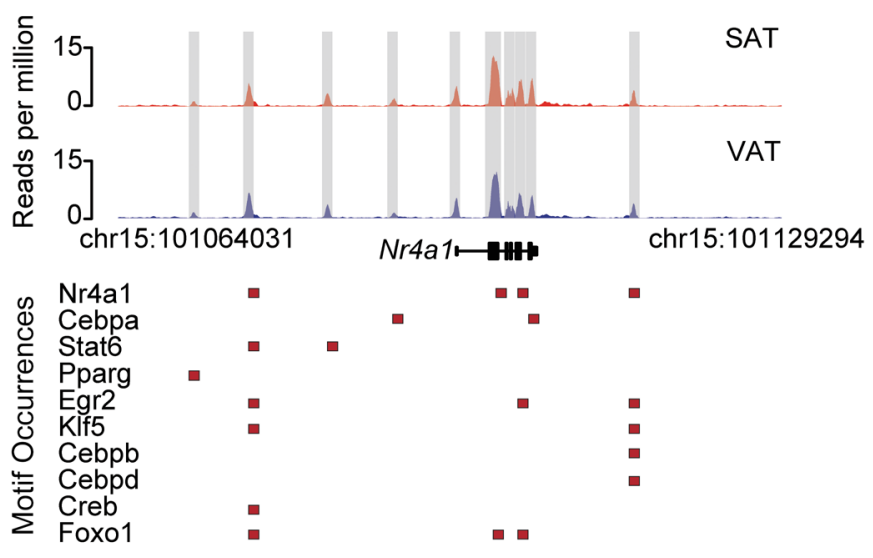

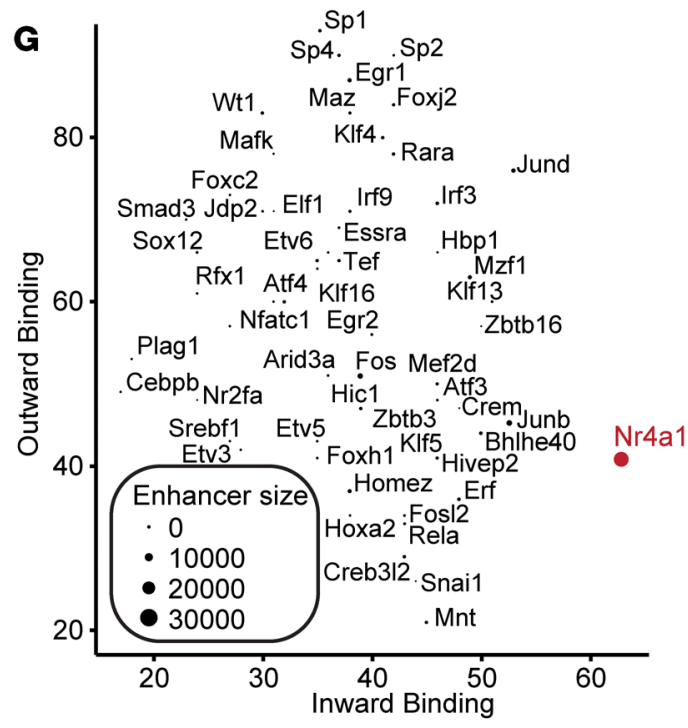

H

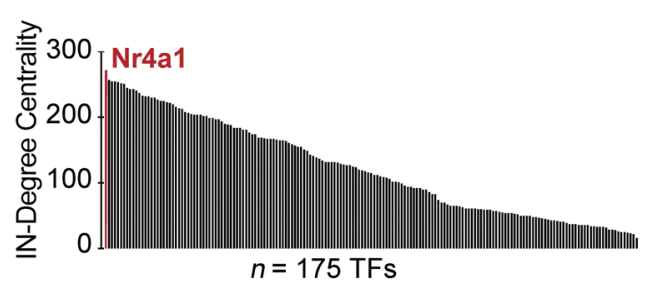

I

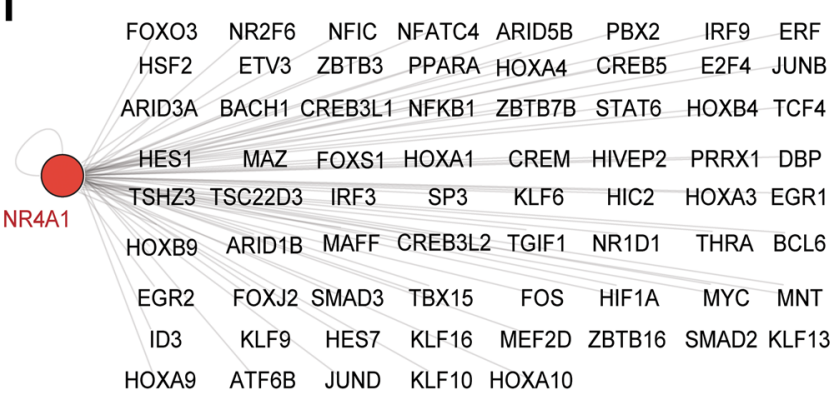

Figure 2. NR4A1 is a constituent of AP core transcriptional circuitry. (A) Bar plot of genomic distribution of ATAC-seq enrichment in APs expressed as a function of the distance to the TSS. (B) Pie chart of overlap between ATAC peaks in APs compared with DNase hypersensitivity maps generated in the mouse ENCODE project. (C) Consensus sequences for the most highly enriched de novo DNA sequence motifs present in unique ATAC peaks in APs. The motifs were then matched to the known TF motif that is closest in sequence composition to the discovered motifs. (D) All enhancers detected in APs ranked by increasing ATAC DNA length in $\mathrm{kb}$. Enhancers are defined as regions of enrichment not fully contained within $\pm 2.5 \mathrm{~kb}$ from a TSS. (E) Gene track of ATAC-seq signal (reads per million) at Nr4a1 locus in APs from SAT and VAT (see also Supplemental Figure 2B). Discrete regions used for motif search are highlighted in gray. (F) The presence of motif occurrences of selected TFs within an ATAC-seq hypersensitivity site (shaded gray in E) are indicated by red boxes. (G) Representative scatter plot of TFs present in the CRC comparing inward and outward binding. Circled size is scaled to represent the size of the largest enhancer regulating a given TF. (H) Bar graph displaying average IN-degree binding (IN-ward centrality) of the top 175 TFs. (I) A subnetwork is shown that includes all super-enhancer-regulated TFs that contain Nr4a1 motifs within their super-enhancers.

ation of AT depots, which yielded putative APs from the inguinal (SAT) and perigonadal (VAT) depots of several mice in approximately 30 minutes. The isolated cell population was enriched in surface markers utilized in more time-intensive multi-marker flow cytometry-sorting strategies, such as CD24, CD29, and Sca-1 (12) (Supplemental Figure 1E). In culture, the cells demonstrated robust differentiation into lipid-laden adipocytes upon exposure to a standard adipogenic cocktail, consisting of dexamethasone, insulin, and 3-isobutyl-1-methylxanthine (DMI), confirming their adipogenic potential (Supplemental Figure 1, F and G).
Having established a rapid method for isolation of primary APs, we aimed to identify transcriptional regulators of AP quiescence. Comparative analyses of the transcriptomes of APs relative to unfractionated AT specimens from the same mice revealed 2,188 and 1,853 genes, which were significantly enriched in APs relative to whole AT $(P<0.01)$. Of the top 150 genes in each depot, only 76 were shared (Figure 1, C and D); however, a gene ontology analysis revealed functional commonality related to cell transcription and proliferation (Figure 1E). When narrowed to genes coding for transcription factors (TF) (13), the overlap was more striking. Of the 22 
A

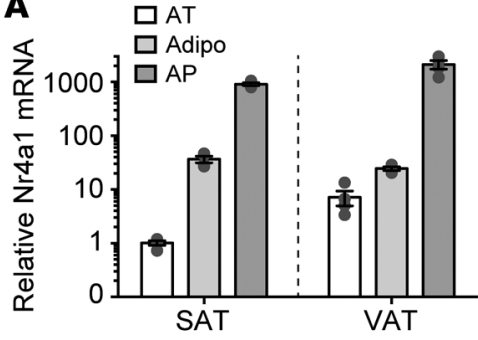

B

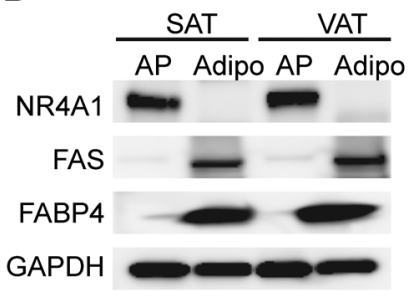

Figure 3. NR4A1 is constitutively expressed in APs. (A) qPCR analysis of Nr4a1 expression in AT fractions: adipocytes (Adipo), APs, and unfractionated AT. Data expressed as bar graphs of mean \pm SEM, normalized to SAT AT, and with individual data points shown as dots. $n=4$. (B) Western blots of AP isolate versus adipocyte fraction. NR4A1 protein expression is compared relative to adipocyte markers FAS and FABP4.
TFs on the 2 lists, 17 were shared between APs from SAT and VAT (Figure 1D). Moreover, quantitative PCR (qPCR) demonstrated all 22 TFs to be concordantly enriched in APs from both depots (Figure $1 F)$, in contrast with genes related to downstream terminal adipocyte differentiation, which were enriched in unfractionated AT (Figure $1 G)$. Our list of enriched TFs contained several "early immediate" response genes, which can be transcribed rapidly in response to stress, raising the question of whether the higher degree of expression of these TFs occurred as a result of the isolation protocol. To address this question, we performed tissue and cell isolations in the presence of an inhibitor of RNA synthesis (actinomycin D) to block new transcription during isolation and observed that the AP fraction remained enriched in these early immediate TFs (Supplemental Figure $1 \mathrm{H}$ and Supplemental Table 1).

Our analysis of freshly isolated primary APs implicates a network of TFs that are distinct from those seen in prior studies of preadipocyte cell lines (14-17). Such differences may reflect inherent programmatic differences between cell lines and the in vivo lineage, as suggested by the distinct epigenomic landscape of primary adipocytes relative to 3T3L1-derived adipocytes $(18,19)$. Our list of enriched TFs is also distinct from prior analyses of primary cell isolates $(17,20,21)$. Beyond differences arising from the specific, genetically encoded markers used for cell selection ( $Z f p 423$ and Pparg), technical differences in cell preparation may also be relevant. For example, profiling performed on $Z f p 423$-expressing committed preadipocytes was completed after an ex vivo culture step (21). Similarly to what occurred in our study, transcriptional profiling of PPARG expression-committed preadipocytes was performed on cells immediately after flow cytometric sorting. Although the resultant list of top genes was distinct from our TF list, transcriptional data from the 2 studies were directionally concordant (Supplemental Table 2). It is also notable that several of the highly expressed TFs in our APs, such as Atf3, Egr1, and members of the Ap-1 and $N r 4 a$ families, have previously been shown to be dynamically upregulated in the early mitotic clonal expansion phase during differentiation of preadipocyte cell lines and in some cases to have antiadipogenic properties (22-27).

NR4A1 is a constituent of the AP core transcriptional circuitry. During development, a small set of TFs establish cell-type-specific gene expression programs by forming core transcriptional regulatory circuitries (CRCs); furthermore, stretch or super-enhancers - large cis-regulatory regions densely bound by TFs and other coactivators - drive the expression of these core TFs (28). We hypothesized that CRC analysis could be used in conjunction with gene expression profiling data to nominate specific TFs for additional functional studies of the AP cell state. To accomplish this analysis, we utilized the assay of transposase-accessible chromatin coupled to massively parallel sequencing (ATAC-seq) to identify putative enhancers in APs. This assay was chosen for 2 reasons: (a) limiting the number of primary APs $\left(\sim 2 \times 10^{5}\right)$ from individual depots renders conventional ChIP assays infeasible, while ATAC generates data with as few as $1 \times 10^{4}$ cells (29); and (b) there is a precedent of statistical correlation between enhancers measured by histone methylation or acetylation (e.g. ChIP-seq for H3K4me1 or H3K27ac) and ATAC-seq in other contexts (30). Biological replicates of ATAC-seq data from SAT or VAT demonstrated statistical reproducibility (Supplemental Figure 2A). Over 83,000 regions of open chromatin were identified. ATAC enrichment was evident at promoters $(10 \%)$; however, more than half of the regions were detected at intergenic or intragenic loci more than $100 \mathrm{~kb}$ from transcriptional start sites (TSS) (Figure 2A). A comparative analysis with publicly available DNase accessibility data from the ENCODE consortium identified 3,834 unique regions (4.6\%) of open chromatin in APs (Figure 2B). Motif analysis of TF-binding sites in these unique ATAC loci revealed enrichment for SOX, CEBP, MAF, and NR2/4 consensus sequences (Figure 2C and Supplemental Figure 2B). We then identified stretch enhancers by the stitching of proximal ATAC peaks and ranking of enhancers by length. This analysis identified 940 stretch enhancers in both SAT and VAT (Figure 2D). Notably, stretch enhancers were evident in proximity to multiple genes encoding regulatory TFs that were also identified in the transcriptional analysis (Figure 1E), including Nr4a1, Jund, Fos, and Klf, suggesting these particular TFs may constitute part of the CRC in APs (Figure 2, D and E and Supplemental Figure 2C). In APs from VAT and SAT, the top-ranked stretch enhancer occurred in proximity to the Nr4a1 gene. Consistent with prior work characterizing super and stretch enhancers (31), the ATAC-seq peaks at the $\mathrm{Nr} 4 \mathrm{a} 1$ locus possessed dense clusters of TF-binding site motifs, including NR4A1 (Figure 2F). We then applied CRC analysis using the top 1,000 enhancer elements to build a network modeling transcriptional regulation in APs. With this network architecture, we then quantified the factors predicted to regulate the expression of a given TF (IN degree) as well the number of enhancers bound by a given TF (OUT degree) (Supplemental Figure 2D). We ranked TFs by their IN/OUT degree of binding to determine their overall interaction network in APs (Figure 2G). In addition to being the TF associated with the top-ranked stretch enhancer, NR4A1 was predicted to have a high degree of connectivity within this TF network, including being the highest ranked TF with respect to IN-degree binding (Figure 2, G-I). This pattern has been observed in the super-enhancers of master-regulatory TFs, such as Myc and the master embryonic stem cell factors Oct4, Sox2, and Nanog, which are characterized by high IN degree binding that establishes robust autoregulatory feedback loops $(32,33)$. As a result of this regulatory 
A
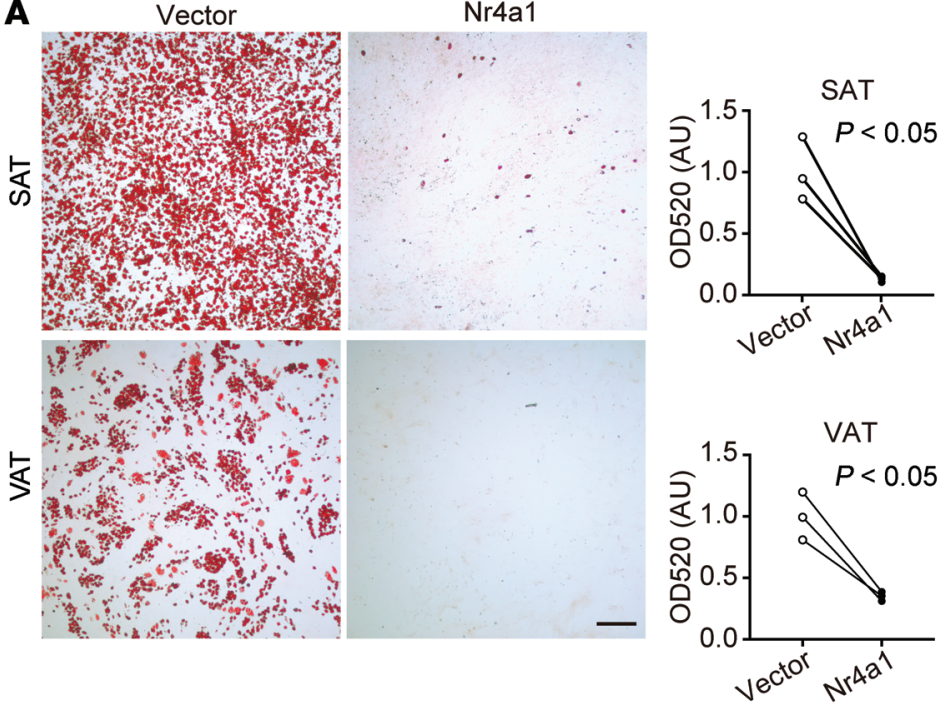

C
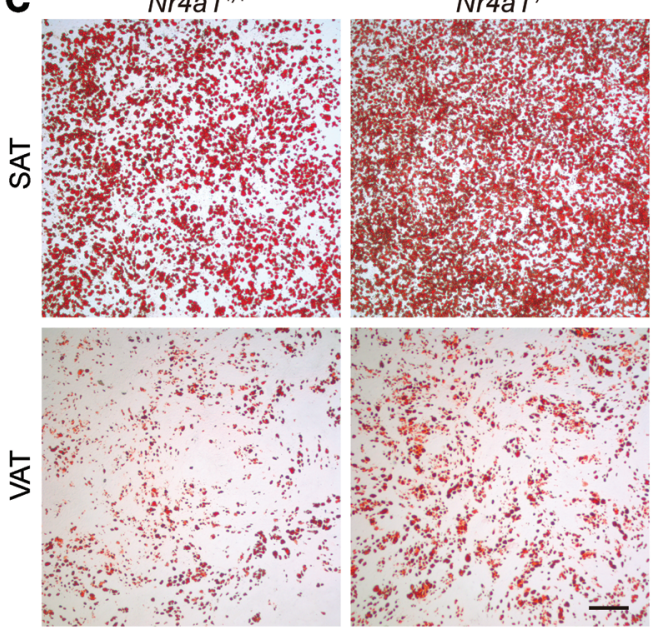

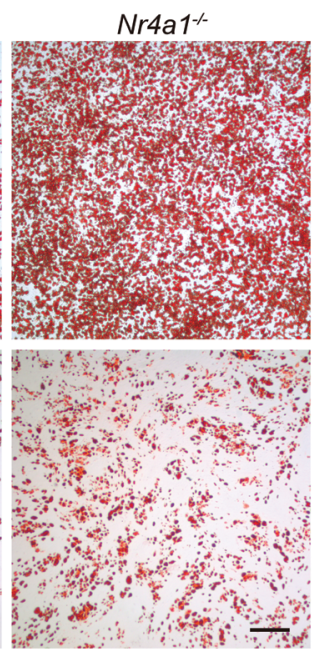

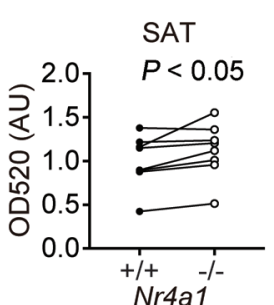

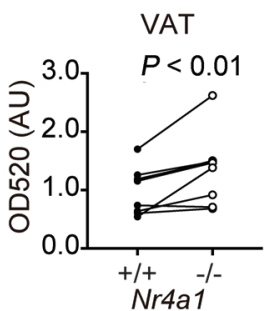

B

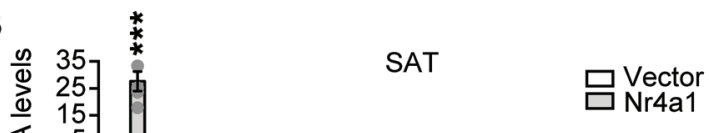

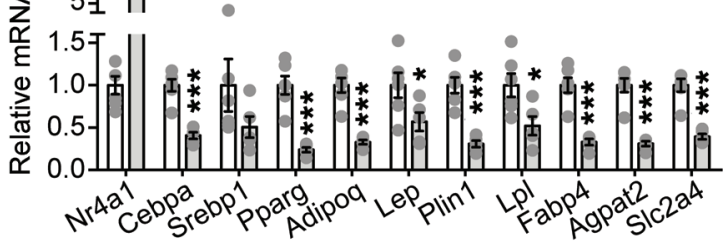

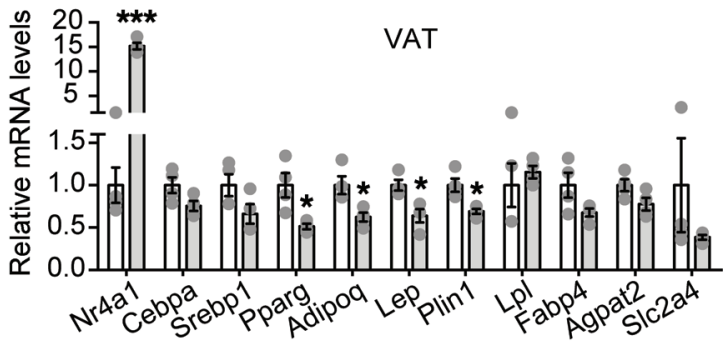

D
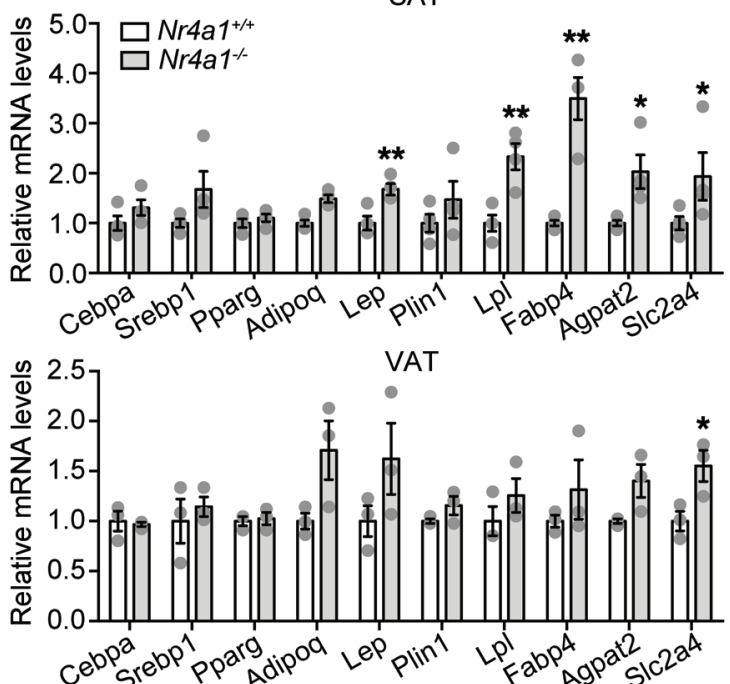

Figure 4. NR4A1 regulates AP differentiation in vitro. (A) ORO staining after induction of adipogenesis in primary APs isolated from SAT and VAT and transduced with retroviral vectors driving overexpression of Nr4a1 (MSCV-Nr4a1) or vector control (MSCV-vector). Data collected after 8 days of adipogenic differentiation. Relative quantification at $520 \mathrm{~nm}$ absorbance after ORO extraction was normalized to MSCV-vector control and expressed as mean of technical replicates. Three independent experiments are shown. Significance was assessed by 2-tailed, paired $t$ test. Scale bar: $1 \mathrm{~mm}$. (B) qPCR analysis of relative expression of $\mathrm{Nr} 4 a 1$ and late adipogenic genes after $\mathrm{Nr} 4 a 1$ overexpression as in $\mathbf{A}$. Data displayed as bar graphs \pm SEM with dots showing individual values. $n=5-6$ technical replicates. ${ }^{*} P<0.05 ;{ }^{* *} P<0.001$, 2-tailed unpaired $t$ test. (C) ORO staining after adipogenic differentiation of APs isolated

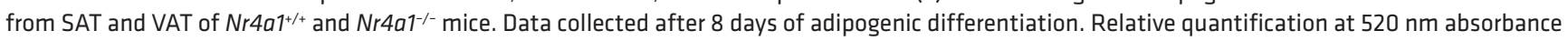

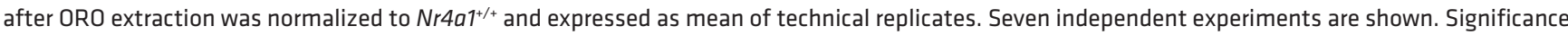
was assessed by 2-tailed, paired $t$ test. Scale bar: $1 \mathrm{~mm}$. (D) qPCR analysis of late adipogenic genes in AP isolated from SAT and VAT of $\mathrm{Nr}_{4} \mathrm{a}^{+/+}$and $\mathrm{Nr} 4 \mathrm{a1}^{-1-}$ mice after induction of adipogenesis. Data displayed as bar graphs \pm SEM with dots showing individual values. $n=3-4$ technical replicates. ${ }^{*} P<0.05 ;{ }^{* *} P<0.01$, 2-tailed unpaired $t$ test.

activity, key transcriptional programs that control cell identity are maintained. Collectively, these data identify NR4A1 as a candidate central node of the CRC in APs (Figure 2, H and I).

Nr4a1 is a constitutively expressed transcriptional regulator of AP differentiation in vitro. We selected the orphan nuclear receptor NR4A1 for additional functional study, given its constitutive expression in APs (Figure 1), and CRC analysis, which predicted a central role for this TF in AP cell state (Figure 2). Although NR4A1 has previously been implicated as a regulator of adipogen- esis in 3T3-L1 cells, prior studies reported absence of expression in resting 3T3-L1 cells and dynamic induction of expression after stimulation of adipogenesis (14). Therefore, we examined a previously unappreciated role for NR4A1 as a constitutively expressed regulator of AP quiescence. We first confirmed high expression of Nr4a1 in freshly isolated APs relative to the adipocyte fraction at the mRNA and protein levels (Figure 3, A and B; and Supplemental Figure 3A). During ex vivo expansion of APs, Nr4a1 was downregulated (approximately 22-fold in SAT, 120 -fold in VAT), then 

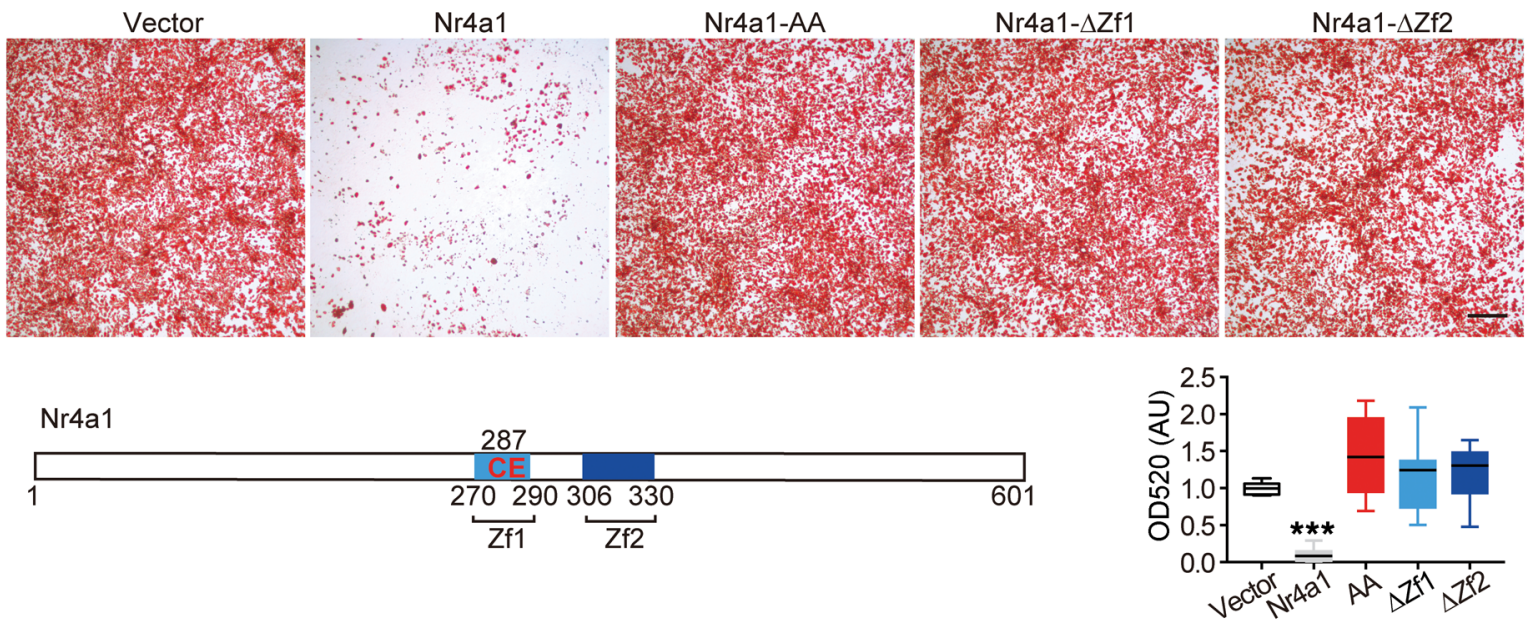

Figure 5. NR4A1 regulation of adipogenesis is dependent on an intact DBD. ORO staining after retroviral overexpression of WT Nr4a1 or DBD mutants (shown in schematic, bottom left) during expansion and differentiation of AP from $\mathrm{Nr}_{4} \mathrm{a}^{-/-}$mice. Data collected after 8 days of adipogenic differentiation. Scale bar: $1 \mathrm{~mm}$. Bottom right shows relative quantification at $520 \mathrm{~nm}$ absorbance after ORO extraction was normalized to MSCV-vector control and expressed as mean of technical replicates. Three independent experiments are shown as box (with mean line) and whiskers (minimum to maximum). Significance was assessed by 1-way ANOVA with Tukey's adjustment for multiple comparisons. ${ }^{* *} P<0.001$.

reexpressed after stimulation with an adipogenic cocktail (DMI) (Supplemental Figure 3B). We detected NR4A1 by immunoblot in unfractionated AT (Supplemental Figure 3C). To more precisely localize NR4A1 in AT, we performed immunofluorescence staining and confocal microscopy (Supplemental Figure 3D). NR4A1 localized to isolectin-positive blood vessels and to perivascular cells that positively stained for the AP marker PDGFR $\alpha$. These data suggest high constitutive expression of NR4A1 in APs, but also confirm promiscuous expression in multiple cell types (34-39). We also investigated whether $\mathrm{Nr} 4 \mathrm{a} 1$ expression was dynamically regulated in AT in vivo in physiologically relevant contexts. We found that the expression of Nr4a1 increased as a function in age in unfractionated SAT and APs isolated from SAT (Supplemental Figure 3, E and F). It was also downregulated in unfractionated AT with high-fat feeding of adult mice, but unaffected by treatment with rosiglitazone (Supplemental Figure 3, G and H).

To elucidate functional significance in APs, we performed Nr4a1 genetic gain and loss of function in ex vivo adipogenesis assays. As previously observed in 3T3L1 cells, overexpression of Nr4a1 during primary AP expansion and adipogenic differentiation attenuated the formation of adipocytes, as measured by Oil Red O (ORO) staining (Figure 4A) (23). The observed decrease in expression of markers of adipocyte differentiation further supports the antiadipogenic effect of NR4A1 (Figure 4B). In contrast, APs isolated from $\mathrm{Nr}_{4} \mathrm{al}^{-/}$demonstrated enhanced adipocyte differentiation upon exposure to DMI relative to WT APs, as assessed by ORO staining and qPCR analysis of gene markers of differentiated adipocytes (Figure 4, C and D). Prior studies observed attenuation of adipogenesis in 3T3-L1 cells with Nr4a1 knockdown in contrast with our findings in primary APs $(40,41)$. Therefore, we also tested loss of function in 3T3-L1 cells. We observed a similar subtle augmentation of adipogenesis in 3T3-L1 cells in which NR4A1 loss of function was achieved by transduction of an Nr4a1-targeted shRNA (Supplemental Figure 4, A and B). We also created a 3T3-L1 line in which $N r 4 a 1$ was functionally delet- ed and observed enhancement of adipogenesis after adipogenic induction (Supplemental Figure 4, C and D). One possible explanation for the discrepancy between our findings and prior studies with 3T3-L1 cells is that the Nr4a1 loss-of-function effect is subtle relative to the inhibitory effect observed with $\mathrm{Nr} 4 a 1$ overexpression. Collectively, Nr4a1 gain- and loss-of-function experiments conducted in primary APs suggest that NR4A1 is a constitutively expressed inhibitor of adipogenesis.

NR4A1 functions through DNA-dependent and DNA-independent mechanisms (42-45). To determine whether the antiadipogenic effects of NR4A1 were mediated through its interaction with DNA, we engineered mutants with substitutions of 2 amino acids in the DNA binding domain (DBD) (287C 288EAA) (46) or with deletions of the first (Zf1) or second (Zf2) zinc fingers. We transduced WT Nr4a1 or the DBD mutants to APs isolated from $\mathrm{Nr} \mathrm{al}^{-/}$mice. Restoration of WT Nr4a1 potently inhibited adipogenesis, an effect that was abrogated when APs were transduced with the DBD mutants (Figure 5). Similar results were observed in 3T3L1 cells (Supplemental Figure 3E). These data establish that NR4A1 negatively regulates adipogenesis through its DBD.

NR4A1 regulates adipogenesis in vivo. We next turned our attention to the $\mathrm{Nr} 4 \mathrm{a}^{-/}$mouse to assess the consequences of NR4A1 loss of function in APs in vivo. We studied juvenile mice (4 weeks old), reasoning that APs have already entered a putative quiescent state at this age, yet the AT retains some degree of plasticity based on our prior work (9). If NR4A1 controls AP function through its role as a transcriptional regulator, we reasoned that its deletion should alter the AP gene expression program. Through transcriptome profiling (RNA-seq) of freshly isolated APs, we identified a shared set of 154 genes increased in APs isolated from both the SAT and VAT of $\mathrm{Nr} 4 \mathrm{al}^{-/}$mice relative to those isolated from $\mathrm{Nr} 4 \mathrm{al}^{+/+}$mice (adjusted $\mathrm{P}<0.05$; Figure 6, A and B). Genes related to the cell cycle were highly represented in this common list of upregulated genes (Figure 6, A, C, and D; Supplemental Figure $5, A-C$ ). Gene set enrichment analysis (GSEA) identified biolog- 
A

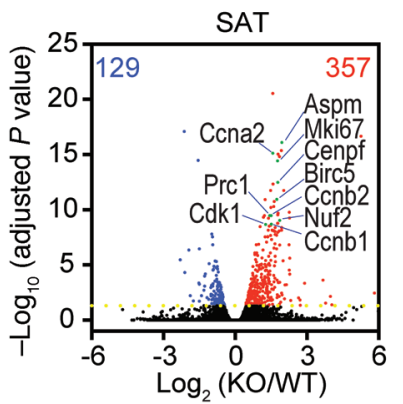

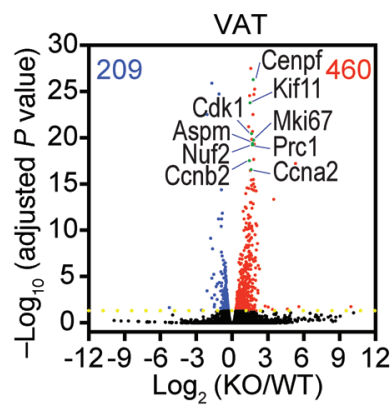

B

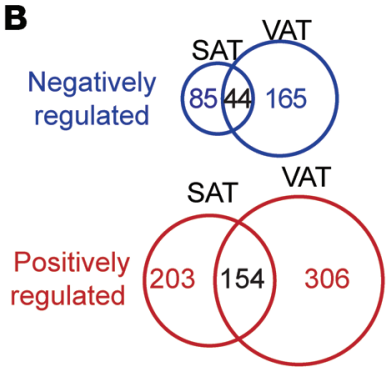

C

Positively regulated

Negatively regulated

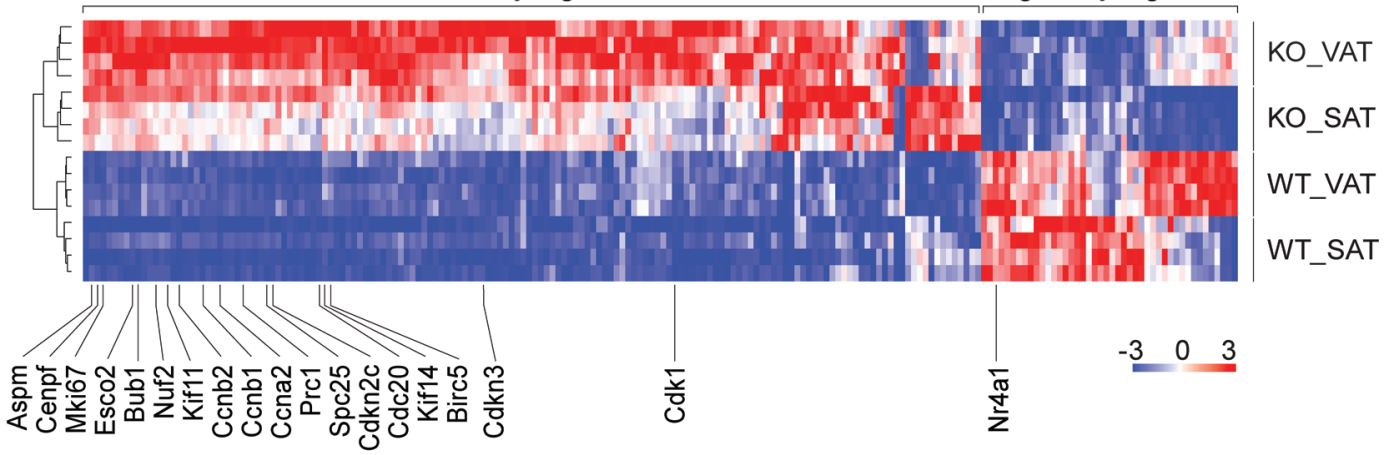

D

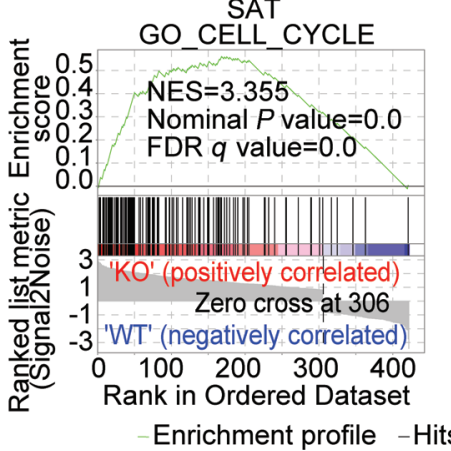

VAT

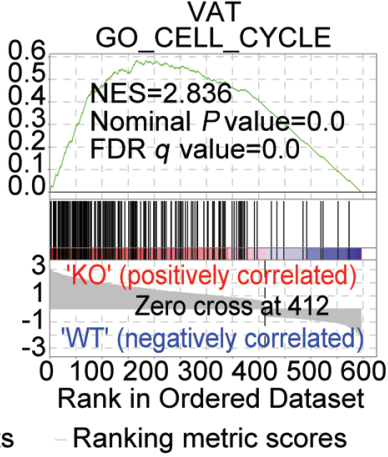

E

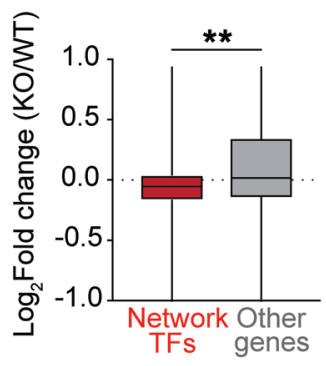

Figure 6. Nr4a1 regulates a cell-cycle transcriptional program. (A) Volcano plots of RNA-seq conducted on AP from $\mathrm{Nr}_{4} \mathrm{a}^{1^{/+}}$(WT) and $\mathrm{Nr} 4 a 1^{-/-}(\mathrm{KO}) \mathrm{mice}$ ( $n=4$ mice). Red dots, positively regulated genes in KO group (adjusted $P<0.05$ ); blue dots, negatively regulated genes in $\mathrm{KO}$ group (adjusted $P<0.05$ ). (B) Venn diagrams show overlap of differentially expressed genes in APs from 2 adipose depots. (C) Hierarchical clustering analysis of common differentially expressed genes from the 2 adipose depots (198 genes). (D) GSEA enrichment plots for GO cell cycle (see also Supplemental Figure 4) in APs from Nr4a1-KO mice $(P<0.001)$ relative to WT. (E) Fold change of all genes in the TF network (Figure 1 ) versus all other expressed genes. TF network genes are as a group downregulated in $\mathrm{KO}$ group relative to all other expressed genes. ${ }^{* *} P<0.01$, Mann-Whitney $U$ test.

ical processes related to cell division as the top-ranked pathways enriched in $\mathrm{Nr}_{4} \mathrm{a}^{--}$APs from both SAT and VAT (Figure 6D, Supplemental Figure 5A). We also leveraged the RNA-seq data to assess whether NR4A1 loss of function would affect other TFs in the AP CRC model. We found a significant global shift in expression of CRC members (Figure 6E), consistent with our model of NR4A1 as a key node in the AP gene-regulatory network.

We selected a panel of differentially regulated cell-cycle genes for additional validation by qPCR and demonstrated higher expression in $\mathrm{Nr}_{4} \mathrm{a1}^{-/}$APs consistent with the RNA-seq data (Supplemental Figure 5D). In the context of DMI stimulation, we found that NR4A1 overexpression inhibited expression of the cell-cycle gene panel (Supplemental Figure 6A). The converse effect was not observed after DMI stimulation of $\mathrm{Nr}^{4} \mathrm{a1^{-/ }}$ APs, consistent with a general theme of more subtle effects with NR4A1 loss of function relative to gain of function (Supplemental Figure 6B). We next used the Nr4a1-null 3T3-L1 line to determine whether knockdown of a panel of candidate NR4A1 effector cell-cycle genes would attenuate the effect of NR4A1 loss of function. Of the 16 genes tested, knockdown of Kif11, Mki67, Prc1, and Spc25 attenuated adipogenesis in Nr4a1-null cells (Supplemental Figure 6C). These data implicate NR4A1 as a repressor of a cell-cycle transcriptional program that is important for adipogenesis.

To determine whether the enhanced cell-cycle gene program affected proliferation in vivo, we performed ${ }^{15} \mathrm{~N}$-thymidine labeling of $\mathrm{Nr} \mathrm{a} \mathrm{1}^{-/ /}$and $\mathrm{Nr} 4 \mathrm{a} 1^{+/+}$mice from weeks 4 to 6 , when AT has entered a more quiescent state. We quantified relative ${ }^{15} \mathrm{~N}$-labeling in the whole stromal vascular fraction rather than the negatively selected AP fraction, since individual AT depots do not reliably yield sufficient APs for isotope ratio mass spectrometry (IRMS) 
A

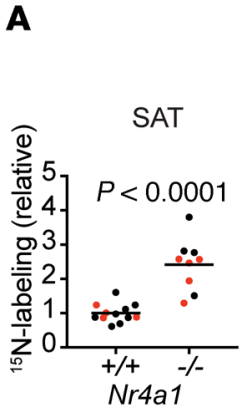

SV

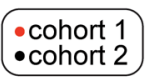

VAT

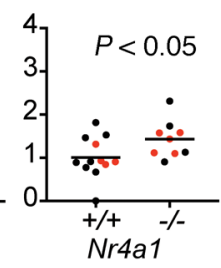

B

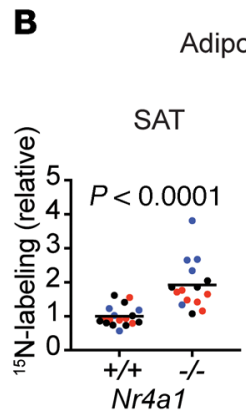

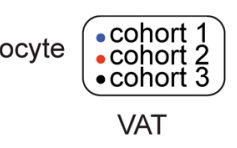

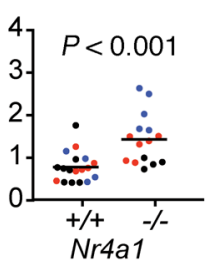

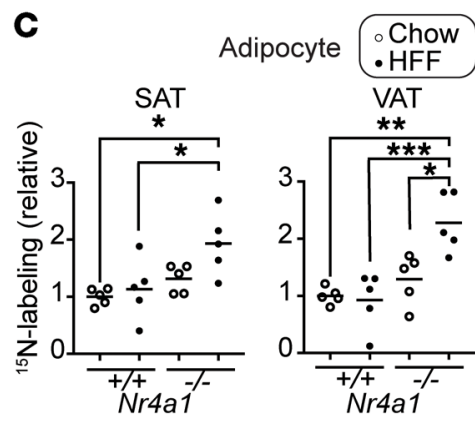

Figure 7. Nr4a1 regulates adipogenesis in vivo. (A) Two cohorts of juvenile (4 weeks old) $\mathrm{Nr} 4 \mathrm{a} 1^{+/+}$and $\mathrm{Nr}^{+} \mathrm{a} 1^{-/-}$male mice labeled for 2 weeks with ${ }^{15} \mathrm{~N}$-thymidine. Relative labeling in stromal vascular cells from SAT and VAT (line indicates mean). Significance was assessed by 2 -tailed, unpaired $t$ test. (B) Three

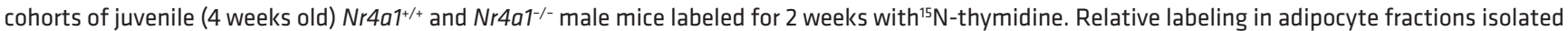

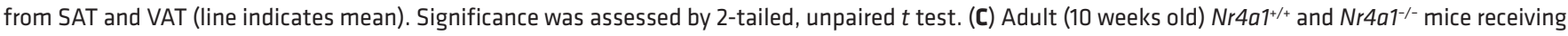
normal chow or high-fat feeding (HFF) were labeled for 8 weeks with ${ }^{15} \mathrm{~N}$-thymidine. Relative labeling in the adipocyte fractions isolated from SAT and VAT (line indicates mean). Two-way ANOVA, Šidák's multiple comparisons adjustment. ${ }^{*} P<0.05 ;{ }^{* *} P<0.01 ;{ }^{* * *} P<0.001$.

analysis. We detected increased ${ }^{15} \mathrm{~N}$-labeling in stromal vascular cells from $\mathrm{Nr}_{4 \mathrm{a1}^{-/-}}$mice, and this effect translated into a concordant increase in ${ }^{15} \mathrm{~N}$-labeling of the adipocyte pool, consistent with augmentation of adipogenesis resulting from NR4A1 loss of function (Figure 7, A and B). These experiments were conducted in male mice; however, we observed no sex-related differences in AT expression of Nr4a1 (Supplemental Figure 7A). A labeling experiment conducted in a mixed cohort confirmed a significant augmentation in SAT adipogenesis (Supplemental Figure 7B). Although the VAT measurements were directionally consistent, they did not meet statistical significance. If AP self renewal and differentiation are coupled in $\mathrm{Nr}_{4} \mathrm{al}^{-/-}$mice, then any proliferative advantage might not translate into an increase in cell number, particularly in young mice. Indeed, a flow cytometric assessment of AP cell-surface-marker expression by stromal vascular cells revealed only a modest increase in $\mathrm{CD} 24^{+}$cells in SAT (Supplemental Figure 7C). Collectively, these data suggest that NR4A1 inhibits adipogenesis in vivo by suppressing AP proliferation.

We have previously demonstrated limited augmentation of adipogenesis in adult murine white AT with obesity (9). We hypothesized that augmentation of AP self renewal through NR4A1 loss of function would facilitate an obesity-mediated AT hyperplastic response. To test this, we labeled adult $\mathrm{Nr}_{4} \mathrm{al}^{-/-}$and $\mathrm{Nr}_{4 \mathrm{al}^{+/+}}$mice with ${ }^{15} \mathrm{~N}$-thymidine during diet-induced obesity. Similarly to what occurred in our prior work, we did not detect an augmentation of ${ }^{15} \mathrm{~N}$-labeling in the adipocyte fractions of WT mice subjected to the obesigenic stimulus. However, when NR4A1 loss of function was coupled with high-fat feeding, we observed a significant increase in adipocyte ${ }^{15} \mathrm{~N}$-labeling consistent with augmented AT plasticity (Figure 7C).

$N R 4 A 1$ regulates AP function and systemic glucose homeostasis in obesity. Our identification of NR4A1 as a constitutive regulator of AP function provided an opportunity to directly test whether augmentation of AT plasticity is metabolically advantageous. However, because NR4A1 has important and potentially confounding functional roles in metabolically relevant tissues, including skeletal muscle, liver, and pancreas (47-49), work with the $\mathrm{Nr} 4 a 1$ global null mouse could not definitively address this hypothesis. To target NR4A1 in APs, we transplanted APs isolated from $\mathrm{Nr}_{4} \mathrm{al}^{-}$
/- or $\mathrm{Nr}_{4} \mathrm{al}^{+/+}$donor mice weekly over the first 3 weeks of high-fat feeding (Figure 8A). After a total of 10 weeks of high-fat feeding, mice administered $\mathrm{Nr}_{4 \mathrm{al}^{-/-}}$cells realized a $59 \%$ increase in body weight, slightly less than the $76 \%$ in mice administered $\mathrm{Nr} 4 \mathrm{al}^{+/+}$ cells (Figure 8B and Supplemental Figure 8A, not significant). We first assessed glucose and insulin tolerance at an early 4 -week time point, finding similar responses in the 2 groups. We repeated testing at 8 and 10 weeks. At these later time points, we observed progressive improvement in both glucose and insulin tolerance in mice administered $\mathrm{Nr}_{4} \mathrm{a1}^{-/-}$APs (Figure 8, C-F). Serum metrics of insulin resistance, including fasting insulin and the homeostasis model for insulin resistance (HOMA-IR) were concordantly improved in mice administered $\mathrm{Nr}_{4} \mathrm{a1}^{-/-}$APs (Figure 8, G-I). We also performed biochemical assays of serum nonesterified free fatty acids (NEFA) and liver triglyceride (TG) content (Figure 8, $\mathrm{J}$ and $\mathrm{K}$ ), and we performed Western blotting of AKT signaling in the liver and skeletal muscle (Supplemental Figure 8B). Under fasting conditions (4 hours) during which the tissues were harvested, we did not observe a change in AKT activity. We did, however, observe a trend toward reduction in serum NEFA and liver TGs in the mice that received $\mathrm{Nr} 4 \mathrm{al}^{-/-}$APs, suggesting the possibility of reduced "spillover" of lipotoxic species as one potential mechanism of improved systemic metabolic function.

An important question raised by our data is, what are the operative mechanisms in the AT depot where the cells are delivered? We hypothesized augmented hyperplasia in the mice receiving $\mathrm{Nr}_{4} \mathrm{al}^{-/-}$APs. Consistent with this, we observed a downward shift in the adipocyte-size distributions in mice receiving $\mathrm{Nr}^{-11^{-/}} \mathrm{APs}$, without a significant difference in depot mass (Figure 8, L and M; and Supplemental Figure 8C). To determine whether this shift was associated with de novo donor-derived adipocytes, we performed PCR on genomic DNA isolated from the subcutaneous adipocyte fractions using $\mathrm{Nr}_{\mathrm{ar}} \mathrm{1}^{-/-}$genotyping primers, detecting evidence of donor genomic DNA in all mice tested (Supplemental Figure 8D). This approach could not provide comparative detection of WT donor APs because the recipients in this group were isogenic (also WT). Therefore, we also used sex-mismatched AP transplantation to assess comparative engraftment efficiencies between $\mathrm{Nr}_{4} \mathrm{a1}^{-/-}$and $\mathrm{Nr}_{4} \mathrm{al}^{+/+}$APs (Supplemental Figure 8E). We 
A

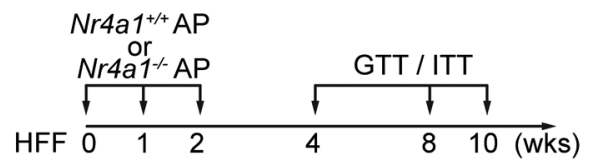

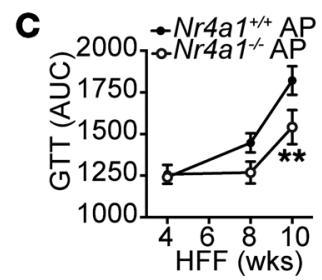

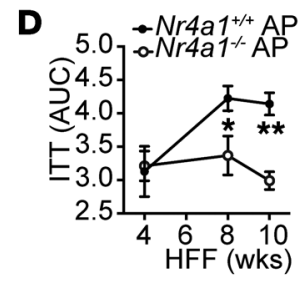

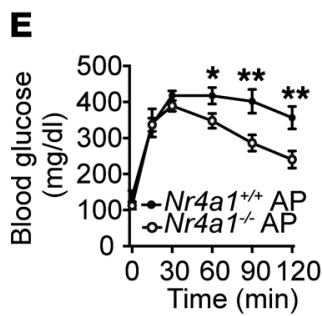

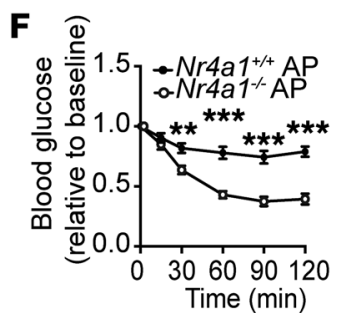

\section{G}

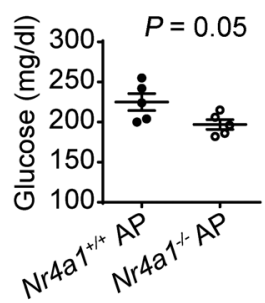

H

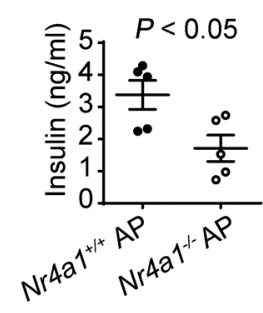

I

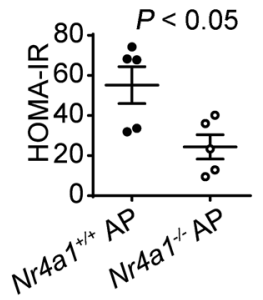

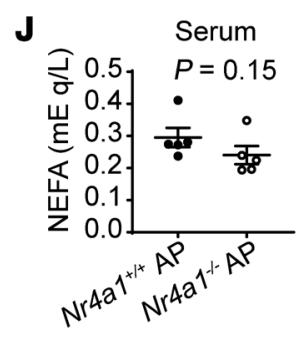
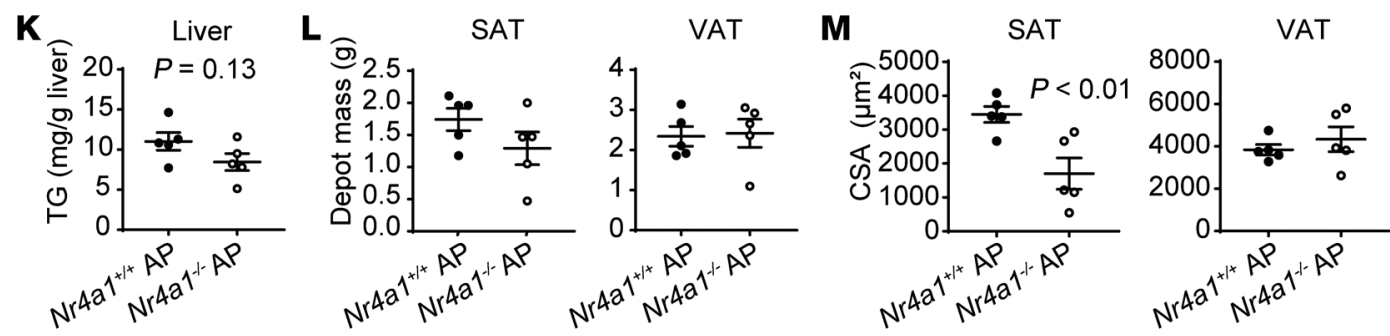

Figure 8. NR4A1 regulates AP function and systemic glucose homeostasis in obesity. (A) Protocol of AP transplantation and high-fat feeding for adult (10 weeks old) C57BL/6 mice. (B) Weight gain expressed as percentage of initial body weight of the mice after $\mathrm{Nr}_{4} a 1^{1^{+/}}$or $\mathrm{Nr} 4 a 1^{1^{-/}} \mathrm{AP}$ transplantation and 10 weeks of HFF. (C) Evolution of the AUC of glucose tolerance test (CTT) during HFF and after AP transplantation. (D) Evolution of the AUC of insulin tolerance test (ITT) during HFF and after AP transplantation. (E) i.p. $(1.5 \mathrm{~g} / \mathrm{kg}) \mathrm{GTT}$ from the 10 -week time point shown in C. (F) i.p. (0.5 U/kg) ITT from the 10-week time point shown in D. (C) Clucose measured after 4-hour fast. (H) Serum insulin levels after 4-hour fast. (I) HOMA-IR derived from glucose/insulin as shown in $\mathbf{H}$ and I. (J) Serum NEFA. (K) Liver TG content. (L) Inguinal (SAT) and perigonadal (VAT) depot weights. (M) Adipocyte mean cross-sectional areas (CSA) for SAT (left) and VAT (right). (B-M) $n=5$, data expressed as mean \pm SEM. Significance was assessed by 2 -tailed, unpaired $t$ test. ${ }^{*} P<0.05$; ${ }^{* *} P<0.01 ;{ }^{* *} P<0.001$.

administered $\mathrm{Nr}_{4} \mathrm{al}^{-/-}$or $\mathrm{Nr} 4 \mathrm{a1^{+/+ }}$ APs isolated from male mice to the contralateral inguinal depots of female recipients. After 2 weeks, we isolated stromal vascular and adipocyte fractions from the inguinal depots and found similar degrees of engraftment in the stromal vascular fraction. At this early time point, we detected an increased percentage of donor-derived adipocytes in the depot receiving $\mathrm{Nr}_{4 \mathrm{a1}^{--}}$APs, consistent with our hypothesis that NR4A1 loss of function programs APs to be more adipogenic.

We performed additional analyses to screen for potential mechanisms by which $\mathrm{Nr}_{4} \mathrm{a1}^{-/}$APs might improve metrics of local AT function. To address the possibility of adipocyte-specific augmentation of glucose utilization with NR4A1 loss of function, we performed glucose-uptake assays on AT explants and in vitro adipocytes differentiated from $\mathrm{Nr}_{4} \mathrm{al}^{-/-}$or $\mathrm{Nr}_{4} \mathrm{al}^{+/+}$mice (Supplemental Figure 8, F and G). We did not observe an increase in glucose uptake in the context of NR4A1 loss of function. We also performed qPCR on the SAT depots in which the cells were injected, testing a panel of genes related to inflammation and metabolic function (Supplemental Figure 8H). We detected a significant increase in $B m p 4, B m p 7$, and $I L-6$, all of which have been implicated as paracrine mediators of brown/beige AT (50-56). Ucp1 expression in SAT samples from the mice receiving $\mathrm{Nr}_{4} \mathrm{a1}^{-/-} \mathrm{APs}$ was also higher, although the results were not statistically signifi- cant. These results raise the intriguing hypothesis that augmentation of adipogenesis results in small adipocytes that provide augmented metabolic and/or paracrine functions. Additional studies will be required to definitively establish the cell-autonomous mechanisms by which AP-targeted NR4A1 loss of function translates into a metabolic advantage in obesity.

\section{Discussion}

In this study, we integrated global gene expression and epigenomic analyses to model the CRC in primary APs. NR4A1 emerged as a strongly connected TF, and functional studies established its role as a negative regulator of adipogenesis, an effect that required an intact DBD. How do these data compare with known studies of NR4A1 biology? Notably, NR4A1 is a predicted CRC node in only 3 other human tissues or cell types (out of 75 tested): heart (left ventricle), pancreas, and brain (middle hippocampus). In each instance, however, the function of NR4A1 differs and includes regulation of growth, apoptosis, or synaptic plasticity, respectively. In addition, global NR4A1 loss of function impairs glucose and lipid metabolism in liver and muscle $(47,48)$. Adding to this complexity, some functions of NR4A1 are independent of DNA binding, such as regulation of apoptosis in gastric carcinoma cells via direct effects in mitochondria (57). In 
this study, the distinct role of NR4A1 as a negative regulator of AP proliferation underscores how TFs collaborate to establish cell state in a highly context-specific manner.

These data suggest that NR4A1 operates as a "gatekeeper" that functionally inhibits adipocyte differentiation through proximal control of AP self renewal. This concept is consistent with both the paradigm of asymmetric stem cell division, whereby a self-renewing cell-division event immediately precedes differentiation of 1 of 2 daughter cells, and with observations that mitotic clonal expansion is a prerequisite to differentiation of cultured preadipocytes (58). Recent evidence suggests that a linear, deterministic model of adipogenesis may be overly simplistic, at least in vivo. Stimulating the adipogenic program with a thiazolidinedione agonist of PPAR $\gamma$ promotes new adipocyte formation coincident with depletion of the AP pool (59). Moreover, we modeled murine adipocyte turnover using data from stable isotope pulse-chase studies as input and found that a subset of adipocytes may arise from differentiation of progenitors without preceding division (9). As such, mediators controlling AP self renewal may operate independently of differentiation pathways. Our data in the adult $\mathrm{Nr} 4 \mathrm{al}^{-/-}$mouse are supportive of this concept. Similarly to our prior work, we did not observe increased ${ }^{15} \mathrm{~N}$-thymidine incorporation in the adipocyte pool of adult mice with high-fat feeding until we coupled high-fat feeding with the cell-cycle-activating effect of NR4A1 loss of function. We propose a model in which a CRC that includes NR4A1 maintains APs in a noncycling quiescent state. This may represent one mechanism that contributes to the tendency of mature AT to expand primarily through hypertrophic rather than hyperplastic growth.

In certain contexts, Nr4a1 functions as an "immediate early response" gene that can be rapidly induced in response to a wide variety of stimuli. The immediate early response program also includes TFs such as $M y c$ and Fos, which are canonical regulators of critical cellular processes, such as cell division, cell survival, and cellular differentiation (60-62). While cellular stress associated with the AP isolation protocol could stimulate this immediate early response signature, our data using multiple orthogonal measurements suggest that NR4A1 is constitutively expressed by progenitors. The mechanism that allows for rapid transcription of immediate early genes is constitutive expression of transcriptional machinery that is poised to initiate transcription in response to kinase-dependent signaling pathways. Many of the cellular stimuli that are known to activate such immediate early response genes may be operative in AT, including low-oxygen tension and inflammation (63-65). Therefore, the concept that AP quiescence is maintained by TFs that are exquisitely sensitive to such microenvironmental stimuli may explain their default tendency to remain quiescent.

It has been hypothesized that obesity represents a paradoxical state of relative lipodystrophy and that type 2 diabetes mellitus (T2DM) ensues when the adipocyte hyperplastic response is insufficient to accommodate the metabolic stress of chronic caloric excess $(10,66-68)$. Consistent with this concept, GWAS have revealed an increase in T2DM risk with genetic variants in adipocyte developmental genes, and quantitative metrics of adipocyte hyperplasia positively correlate with insulin sensitivity (4, $6,69-71)$. In addition, some mouse models demonstrate evidence of enhanced adipocyte hyperplasia coincident with attenuation of the metabolic sequelae of obesity $(72,73)$. It has been challenging, however, to establish a direct causal link between the adipocyte hyperplastic response and a metabolic advantage, in part because of the limited tools available to genetically manipulate AP function in a targeted fashion in vivo, in contrast with the adipocyte-specific Cre-Lox approaches to target terminally differentiated adipocytes. We targeted NR4A1 loss of function to the AP by transplanting $\mathrm{Nr}_{4} \mathrm{a1}^{-/-}$APs into WT recipients. By demonstrating improved glucose metabolism after administration of APs, genetically programmed for adipogenic self renewal and differentiation, we provide direct evidence that augmentation of AT plasticity attenuates the risk of obesity-related diabetes mellitus, just as AP transplantation rescues the metabolic phenotype of lipodystrophic mice (12). Yet our study leaves a number of important questions unanswered. Importantly, are there cell-autonomous mechanisms by which functional APs promote systemic metabolic health? For example, it may be that immature adipocytes in the subcutaneous depot are metabolically hyperactive on the brown/beige fat spectrum or that they secrete beneficial paracrine factors. Alternatively, there may be indirect benefits mediated by the prevention of adipocyte hypertrophy. This study provides conceptual rationale and a candidate molecular approach for further investigating cellular mechanisms underlying the metabolic benefits of adipogenesis in SAT as a step toward therapeutically augmenting AT plasticity in diabetes mellitus.

\section{Methods}

Mice. Mice were maintained on a 12-hour dark/12-hour light cycle at $22^{\circ} \mathrm{C} \pm 2{ }^{\circ} \mathrm{C}$, receiving food and water ad libitum. WT C57BL/6 mice were purchased from either Charles River or Jackson Laboratory. $\mathrm{Nr} 4 \mathrm{al}^{-/-}$breeders were purchased from Jackson Laboratory and maintained on a C57BL/ 6 background. For diet-induced obesity studies, mice were administered a high-fat diet or normal chow control (Research Diets D12492/ D12450J).

Stable isotope tracer methodology. ${ }^{15} \mathrm{~N}$-thymidine (Cambridge Isotope Laboratories) was administered by twice daily subcutaneous injection (25 mg/kg, Figure 1, A and B) or via drinking water (100 $\mathrm{mg} / \mathrm{l}$, Figure 7$)$, as previously described $(9,74)$. Stromal vascular and adipocyte fractions were isolated and analyzed by IRMS, as previously described (9). Briefly, samples were dried in tin cups and introduced to an elemental analyzer (Vario PYRO Cube, Elementar) coupled to an IRMS (Isoprime 100, Elementar). Tuning was confirmed with urea standards, and samples obtained from unlabeled mice were used as an additional control for the natural background ratio $\left({ }^{15} \mathrm{~N} /{ }^{14} \mathrm{~N}=0.37 \%\right)$. ${ }^{15} \mathrm{~N}$-labeling was detected as signal above the natural ${ }^{15} \mathrm{~N} /{ }^{14} \mathrm{~N}$ ratio.

Isolation and culture of APs. Mice used as a source of APs were 4 to 6 weeks old. Perigonadal (visceral) or inguinal (subcutaneous) AT depots were excised, minced, and digested in an enzyme cocktail consisting of collagenase D (Roche) and dispase II (Zenbio), as described previously, similarly to the Rodbell method (75). After centrifugation $\left(4^{\circ} \mathrm{C}, 400 \mathrm{~g}, 10\right.$ minutes), the pelleted stromal vascular fraction was subjected to negative selection by column-based magnetic-assisted cell sorting (MACS) (Miltenyi Biotec) (Supplemental Figure 1C). Monoclonal anti-CD31 microbeads (Miltenyi Biotec) were utilized to negatively select endothelial cells. A biotin-conjugated monoclonal anti-lineage cocktail, followed by anti-biotin MicroBeads (Miltenyi Biotec), was used to negatively select hema- 
topoietic cells. The targeted lineage ${ }^{+} / \mathrm{CD} 31^{+}$cells were then depleted by retaining them on a MACS Column in the magnetic field of a MACS Separator. The unselected cell fraction was eluted through the column. For analyses of freshly isolated APs, the cells were used at this stage. For analyses of cultured APs, the cell isolate was cultured in DMEM-F/12 GlutaMAX medium (Gibco, Thermo Fisher Scientific) with $10 \%$ Premium FBS (Corning) and penicillin/streptomycin. Medium was changed for the first time 2 days after plating.

AP transplantation. The AP transplantation study protocol is as shown in Figure 8A. Cells were isolated from $\mathrm{Nr}_{4} \mathrm{al}^{+/+}$or $\mathrm{Nr}_{4} \mathrm{a1}^{-/-}$ donor mice as described above and expanded in $150 \mathrm{~cm}^{2}$ flasks. $1 \times$ $10^{6}$ Donor cells were injected subcutaneously into the ventral inguinal area of WT C57BL/6 recipient mice. The first dose of cells was administered at age 10 weeks at the onset of high-fat feeding; 2 additional doses were administered at weekly intervals. Comparative donor cell engraftment efficiencies were determined in separated sex-mismatched AP transplantation experiments using a qPCR-based technique (76). Insulin tolerance tests were performed by injecting $0.5 \mathrm{U} /$ $\mathrm{kg}$ regular insulin i.p. after a 4-hour fast. Glucose tolerance tests were performed by i.p. injection of glucose $(1.5 \mathrm{~g} / \mathrm{kg})$ after a 16 -hour fast. Blood was collected from the tail vein and measured by glucometer (Contour, Bayer). Insulin levels in serum were measured by ELISA (EMD Millipore). HOMA-IR was calculated as follows: fasting blood glucose $(\mathrm{mmol} / \mathrm{l}) \times$ fasting serum insulin $(\mathrm{mIU} / \mathrm{l}) / 22.5$. Serum NEFA was measured using an in vitro enzymatic colorimetric assay (Wako Chemicals), with a sensitivity of $0.0014 \mathrm{mEq} / \mathrm{l}$. Liver TG contents were measured by the Colorimetric Assay Kit (Cayman).

Adipogenesis assay. Primary APs or 3T3-L1 cells (ZenBio) were cultured to confluence. All data for this manuscript involved stimulation with the full adipogenic cocktail, containing dexamethasone $(1 \mu \mathrm{M})$, insulin $(10 \mu \mathrm{g} / \mathrm{ml})$, and isobutylmethylxanthine $(0.5 \mathrm{mM})$. APs were cultured in DMEM-F/12 GlutaMAX, 10\% FBS. 3T3-L1 cells were cultured in DMEM GlutaMAX, 10\% FBS. At 96 hours after induction, cells were switched to maintenance medium, consisting of standard culture medium supplemented with insulin $(10 \mu \mathrm{g} / \mu \mathrm{l})$. Maintenance medium was changed every 2 days. Differentiation into mature lipid-containing adipocytes was determined by ORO staining (77) and quantified by measurement of isopropanol-extracted ORO dye at an optical density of $520 \mathrm{~nm}$ (OD520). In a subset of experiments, cells were plated in 96-Well Clear Bottom Black Plates (Corning), lipid droplets stained with fluorescent neutral lipid dye (Cayman), and lipid droplet staining quantified with a SpectraMax M5 plate reader (Molecular Devices). Flow cytometry. PE-conjugated antibodies used for flow cytometry were purchased from eBioscience (CD45-PE, CD24, CD29, Sca-1, CD140a, CD140b, and isotype) and used at a 1:100 dilution. Flow cytometry was performed with an Accuri C6 Analyzer or a BD Aria Sorter (BD Biosciences) and the data analyzed with FlowJo software.

Microarray. Gene expression profiling was performed and analyzed at the Partners Sequencing, Genotyping, Microarray Research Core (Cambridge, Massachusetts, USA), and data were deposited in the NCBI's Gene Expression Omnibus database (GEO GSE118876). For each group, total RNA samples isolated from both progenitor cells and whole tissue from SAT or VAT of 3 individual C57BL/6 mice were labeled and hybridized in an Affymetrix Gene Chip (GeneChip Mouse Gene 2.0 ST Array) according to the manufacturer's protocol. Normalization and statistical analysis of gene expression was performed using Affymetrix GeneChip Operating Software (GCOS), and differentially expressed genes were identified using moderated $t$ statistics with an adjusted $P$ value of less than 0.05. All arrays were normalized and processed in GenePattern (https://software.broadinstitute.org/cancer/ software/genepattern). GO Biological process annotation analysis was performed by DAVID (78).

ATAC-seq. ATAC-seq data processing was completed as reported, with minor variations $(29,79)$, and data were deposited in the GEO database (GSE118856). In brief, Bowtie2 was used to align sequencing reads to the $\mathrm{mm} 9$ genome build. MACS2 narrowPeak was used to call peaks for ATAC-seq. Comparisons of genomic loci were performed with BEDOPS (80), and mapping reads to genomic loci was performed with Bamliquidator (https://github.com/BradnerLab/pipeline/wiki/bamliquidator). Enhancer identification was carried out using ROSE2 (https:// github.com/BradnerLab/pipeline). Network construction utilized the Coltron Python package (https://pypi.python.org/pypi/coltron).

Software. The following versions were used: MACS, version 2.1; Bowtie2, version 2.2.9; BEDOPS, version 2.4.19; Bamliquidator, version 1.2.0; Samtools, version 0.1.19; FIMO, version 4.91; and NetworkX, version 1.8.1.

Sequencing analysis. Raw paired-end sequencing data were mapped to the $\mathrm{mm} 9$ build of the human genome with Bowtie 2 with default settings and the parameters $-\mathrm{p} 4$ and $-\mathrm{k} 1$ (81). Mapped reads were filtered to remove duplicate reads, with those reads mapping to chromosome $\mathrm{M}$ and to regions in the ENCODE blacklist. Reads mapping to the forward strand were shifted forward $4 \mathrm{bp}$, and reads mapping to the reverse strand were shifted backwards $5 \mathrm{bp}$. MACS2 was then used to find peak default settings.

Super-enhancer definition. The ROSE2 algorithm was used in "stretch" mode for identification of super-enhancers, which included a sample-specific stitching length optimization. The punctate and less continuous nature of the ATAC-seq signal (as compared with the ChIP-seq signal) is not compatible with the classic read-density calculation for super-enhancers, so using enhancer length is a useful surrogate. This has been explored further in Varshney et al. (82).

Peakoverlap analysis. All peaks were merged into a consensus ATAC peak list using the BEDOPS -merge command. These peaks were compared with the ENCODE mm9 master DNase-seq peak list, and overlaps were calculated using the BEDOPS -element-of command.

Gene tracks. Gene tracks were generated with BamPlot_turbo.py (https://github.com/BradnerLab/pipeline) and normalized to reads per million. The number of bins per track was set at 500 bins per genomic region.

Motif enrichment. Motif enrichment in ATAC peaks was carried out with FindMotifsGenome from the HOMER software package.

Transcriptional regulatory networks. This analysis was adapted from Saint-André et al. to utilize information from ATAC-seq and across multiple samples (28). To define the set of TF nodes for the transcriptional regulatory network (TRN), we assigned the top 1,000 genes in the enhancer rank-order list to nearby genes with accessible promoters. Promoters overlapping the broad enhancer region are given priority for assignment, then genes within $100 \mathrm{~kb}$ of the enhancer edges. If neither condition is met, the enhancer is assigned to the closest promoter. Then, discrete ATAC peaks within the large enhancer domains were considered. The underlying sequence from these peaks was extracted, and FIMO was used to search for binding sites of node TFs in these peaks with a $P$ value of $1 \times 10^{-4}$. TF position-weight matrices were taken from TRANSFAC and JASPAR (83- 
85). When a motif was identified in an enhancer, an edge was drawn in the network between the node whose motif was present and the node that was regulated by the enhancer. The most highly connected TFs were plotted in Cytoscape (86).

RNA-seq. RNA quantification, library construction, sequencing, and data analysis were performed at the Center for Cancer Computational Biology Core Facilities at the Dana Farber Cancer Institute, and data were deposited in the GEO database (GSE118879). Purification of total RNA isolated from APs of $\mathrm{Nr}_{4} \mathrm{al}^{+/+}$and $\mathrm{Nr} 4 \mathrm{a1}^{-/-}$mice was performed using an RNeasy Micro Kit (QIAGEN). Genomic DNA was removed using the DNA-Free DNA Removal Kit (Ambion). RNA was analyzed by the Qubit RNA BR Assay Kit (Thermo Fisher Scientific) according to the manufacturer's instructions. Sequencing libraries were prepared using the NEBNext Ultra RNA Library Prep Kit (NEB). RNA-seq experiments were run using single-read 75 flow cells on an Illumina NextSeq 500 platform. Sequencing reads were aligned to the reference genome using the RNA-specific STAR aligner. Quality control testing and verification involved postsequencing assessment with FastQC (S. Andrews, Babraham Bioinformatics; http://www.bioinformatics.babraham.ac.uk/projects/fastqc) and with RNA-SeQC following sequence alignment (87). FeatureCounts was used to count sequencing reads, which mapped to the reference genome at the gene level (88). Counts were subsequently normalized between samples by DESeq (89). RUV-Seq was utilized to normalize sequencing reads, which mapped to a reference genome of spike-in gene sets (ERCC RNA Spike-in Mix, Ambion). Differential expression analysis was performed between the desired experimental contrasts for both normalization methods independently using the DESeq software suite (89). GSEA was performed on the Broad GSEA algorithm platform (90) (http://software.broadinstitute.org/gsea/index.jsp). For RNA fold-change analysis of TFs within the super-enhancer transcriptional network, we considered all TFs regulated by super-enhancers and compared the fold change of these genes to all other expressed genes (fragments per kb million [FPKM] > 1). Distributions were compared with the Mann-Whitney $U$ test.

Plasmids. Nr4a1 CDS (OriGene) was subcloned into retroviral vector MSCV-PIG (Addgene). Nr4a1 DNA-binding domain mutants were generated using the NEB Q5 Mutagenesis Kit (NEB). For Nr4a1 loss of function, validated shRNA (MilliporeSigma) or scramble sequences were subcloned into a retroviral vector (pMKO.1). Knockdown efficiency was confirmed by qPCR in 3T3-L1 cells (Supplemental Figure 3A). shRNA TRC numbers were as follows: Nr4a1, TRCN0000234021; Aspm, TRCNO000110639; Bub1, TRCNO000344980; Birc5, TRCN0000054613; Ccnb1, TRCNO000332716; Cdk1, TRCN0000274559; Cenpf, TRCNO000241993; Cdkn3, TRCNO000454139; Cdc20, TRCNO000280230; Ccnb2, TRCNO000077771; Ccna2, TRCN0000077764; Esco2, TRCN0000077181; Kif11, TRCNO000091774; Mki67, TRCNO000238590; Nuf2, TRCNO000244489; Prc1, TRCN0000352509 ; and Spc25, TRCNOO00329276.

qPCR. Total RNA was isolated from tissues or cells using TRIzol (Invitrogen). cDNA was synthesized using the High-Capacity cDNA Reverse Transcription Kit (Life Technologies) from $1 \mu \mathrm{g}$ RNA. Real-time qPCR was performed with Power SYBR Green PCR mixture (Applied Biosystems) on the QuantStudio 5 Real-Time PCR System (Applied Biosystems). Gene expression was normalized to $18 \mathrm{~S}$ rRNA or GAPDH. The $\Delta \Delta \mathrm{Ct}$ method was used to calculate the fold change in transcript levels. Primer sequences are provided in Supplemental Table 3.
Immunofluorescence imaging. AT specimens for confocal imaging were fixed and processed following published protocols $(91,92)$. ATs were labeled with anti-NR4A1 (Novus, NB100-56745), anti-CD140a (PDGFR $\alpha$, Thermo Fisher 14-1401-82), anti-isolectin (Isolectin Alexa Fluor 647 Conjugate, Thermo Fisher, I32450), and DAPI (Thermo Fisher). The tissues were imaged and sectioned at $0.15 \mu \mathrm{m}$ intervals using excitation from a 633 or $543 \mathrm{~nm}$ laser or a $488 \mathrm{~nm}$ line of an argon laser and $\mathrm{a} \times 63,1.2 \mathrm{NA}$ water immersion objective on a laser-scanning confocal microscope (TCS SP5; Leica).

Antibodies/Western blot analysis. Western blot analysis was accomplished using antibodies to GAPDH (Santa Cruz Biotechnology Inc., catalog sc-32233), NR4A1 (Abcam, catalog ab109180), FAS (Cell Signaling Technology, catalog 3180), FABP4 (Cell Signaling Technology, catalog 3544), pAKT-Ser473 (Cell Signaling Technology, catalog 4060), pAKT-T308 (Cell Signaling Technology, catalog 13038), and AKT (Cell Signaling Technology, catalog 4691). Chemiluminescent blot images were captured by the Image Quant LAS 4000 system (GE Life Sciences) and quantified using Quantity One (Bio-Rad). See complete unedited blots in the supplemental material.

Glucose-uptake assay. APs isolated from $\mathrm{Nr}_{4} \mathrm{al}^{+/+}$and $\mathrm{Nr} 4 \mathrm{a}^{-/-}$ mice were differentiated into adipocytes. Subcutaneous fat pad explants $(20 \mathrm{mg})$ from $\mathrm{Nr} 4 \mathrm{al}^{+/+}$and $\mathrm{Nr}^{+\mathrm{a}^{-/-}}$mice were dissected excluding visible connective tissue and blood vessels. Glucose uptake of differentiated APs in vitro and SAT explants ex vivo was measured by the Glucose Uptake-Glo Assay Kit (Promega) following the manufacturer's protocol and a previous report (93). Glucose uptake was assayed with or without insulin stimulation (100 ng/ $\mu \mathrm{l})$ for 30 minutes. ORO staining was utilized to determine relative mature adipocyte counts in parallel.

Statistics. Statistical analyses were performed using Prism 7 (GraphPad). A Shapiro-Wilk test was used to assess normality. For normally distributed data sets, an unpaired, 2-tailed $t$ test was performed to compare experimental with control groups. For nonnormally distributed data, a Mann-Whitney $U$ test was used. A 2-sided $P$ value of less than 0.05 was considered significant.

Study approval. All animal experiments were approved by and in compliance with the Brigham and Women's Hospital Institutional Animal Care and Use Committee.

\section{Author contributions}

YZ and MLS conceived the study. YZ, SK, ML, JPO, DX, JDB, and MLS performed experiments. YZ and AJF analyzed the data. JDB reviewed and edited the manuscript. YZ and MLS wrote the original draft. MLS supervised the experiments. MLS acquired funding.

\section{Acknowledgments}

This work was funded by NIH/National Institute of Diabetes and Digestive and Kidney Diseases (K08DK090147 and R03DK106477 to MLS) and the Gilead Research Scholar Award (to MLS). The authors thank Yiwei Li for advice regarding immunostaining.

Address correspondence to: Matthew L. Steinhauser, 77 Avenue Louis Pasteur, New Research Building 458C, Boston, Massachusetts 02115, USA. Phone: 617.525.4716; Email: msteinhauser@ bwh.harvard.edu. 
1. Nguyen NT, Nguyen XM, Lane J, Wang P. Relationship between obesity and diabetes in a US adult population: findings from the National Health and Nutrition Examination Survey, 19992006. Obes Surg. 2011;21(3):351-355.

2. Mokdad AH, et al. Prevalence of obesity, diabetes, and obesity-related health risk factors, 2001. JAMA. 2003;289(1):76-79.

3. Garg A. Acquired and inherited lipodystrophies. NEngl JMed. 2004;350(12):1220-1234.

4. Majithia AR, et al. Rare variants in PPARG with decreased activity in adipocyte differentiation are associated with increased risk of type 2 diabetes. Proc Natl Acad Sci U S A. 2014;111(36):13127-13132.

5. Majithia AR, et al. Prospective functional classification of all possible missense variants in PPARG. Nat Genet. 2016;48(12):1570-1575.

6. Chu AY, et al. Multiethnic genome-wide meta-analysis of ectopic fat depots identifies loci associated with adipocyte development and differentiation. Nat Genet. 2017;49(1):125-130.

7. Spalding KL, et al. Dynamics of fat cell turnover in humans. Nature. 2008;453(7196):783-787.

8. Guillermier C, et al. Imaging mass spectrometry demonstrates age-related decline in human adipose plasticity. JCI Insight. 2017;2(5):e90349.

9. Kim SM, et al. Loss of white adipose hyperplastic potential is associated with enhanced susceptibility to insulin resistance. Cell Metab. 2014;20(6):1049-1058.

10. Wang QA, Tao C, Gupta RK, Scherer PE. Tracking adipogenesis during white adipose tissue development, expansion and regeneration. Nat Med. 2013;19(10):1338-1344.

11. Greenwood MR, Hirsch J. Postnatal development of adipocyte cellularity in the normal rat. J Lipid Res. 1974;15(5):474-483.

12. Rodeheffer MS, Birsoy K, Friedman JM. Identification of white adipocyte progenitor cells in vivo. Cell. 2008;135(2):240-249.

13. Zhang HM, et al. AnimalTFDB: a comprehensive animal transcription factor database. Nucleic Acids Res. 2012;40(Database issue):D144-D149.

14. Fu M, et al. A nuclear receptor atlas: 3T3-L1 adipogenesis. Mol Endocrinol. 2005;19(10):2437-2450.

15. Burton GR, Nagarajan R, Peterson CA, McGehee RE. Microarray analysis of differentiation-specific gene expression during 3T3-L1 adipogenesis. Gene. 2004;329:167-185.

16. Burton GR, Guan Y, Nagarajan R, McGehee RE. Microarray analysis of gene expression during early adipocyte differentiation. Gene. 2002;293(1-2):21-31.

17. Gupta RK, et al. Transcriptional control of preadipocyte determination by Zfp423. Nature. 2010;464(7288):619-623.

18. Roh HC, Tsai LT, Lyubetskaya A, Tenen D, Kumari M, Rosen ED. Simultaneous transcriptional and epigenomic profiling from specific cell types within heterogeneous tissues in vivo. Cell Rep. 2017;18(4):1048-1061.

19. Mikkelsen TS, et al. Comparative epigenomic analysis of murine and human adipogenesis. Cell. 2010;143(1):156-169.

20. Tang W, et al. White fat progenitor cells reside in the adipose vasculature. Science. 2008;322(5901):583-586.

21. Gupta RK, et al. Zfp423 expression identifies committed preadipocytes and localizes to adi- pose endothelial and perivascular cells. Cell Metab. 2012;15(2):230-239.

22. Boyle KB, et al. The transcription factors Egr1 and Egr2 have opposing influences on adipocyte differentiation. Cell Death Differ. 2009;16(5):782-789.

23. Chao LC, Bensinger SJ, Villanueva CJ, Wroblewski K, Tontonoz P. Inhibition of adipocyte differentiation by Nur77, Nurr1, and Nor1. Mol Endocrinol. 2008;22(12):2596-2608.

24. Stephens JM, Butts MD, Pekala PH. Regulation of transcription factor mRNA accumulation during 3T3-L1 preadipocyte differentiation by tumour necrosis factor-alpha. J Mol Endocrinol. 1992;9(1):61-72.

25. Patel YM, Lane MD. Mitotic clonal expansion during preadipocyte differentiation: calpain-mediated turnover of p27. J Biol Chem. 2000;275(23):17653-17660.

26. Ntambi JM, Young-Cheul K. Adipocyte differentiation and gene expression. J Nutr. 2000;130(12):3122S-3126S.

27. Kim HB, et al. NFATc4 and ATF3 negatively regulate adiponectin gene expression in 3T3-L1 adipocytes. Diabetes. 2006;55(5):1342-1352.

28. Saint-André V, et al. Models of human core transcriptional regulatory circuitries. Genome Res. 2016;26(3):385-396.

29. Buenrostro JD, Giresi PG, Zaba LC, Chang HY, Greenleaf WJ. Transposition of native chromatin for fast and sensitive epigenomic profiling of open chromatin, DNA-binding proteins and nucleosome position. Nat Methods. 2013;10(12):1213-1218.

30. Lara-Astiaso D, et al. Immunogenetics. Chromatin state dynamics during blood formation. Science. 2014;345(6199):943-949.

31. Whyte WA, et al. Master transcription factors and mediator establish super-enhancers at key cell identity genes. Cell. 2013;153(2):307-319.

32. Hnisz D, et al. Convergence of developmental and oncogenic signaling pathways at transcriptional super-enhancers. Mol Cell. 2015;58(2):362-370.

33. Gryder BE, et al. PAX3-FOXO1 establishes myogenic super enhancers and confers BET bromodomain vulnerability. Cancer Discov. 2017;7(8):884-899.

34. Murphy SR, Oslund KL, Hyde DM, Miller LA, Van Winkle LS, Schelegle ES. Ozone-induced airway epithelial cell death, the neurokinin-1 receptor pathway, and the postnatal developing lung. Am JPhysiol Lung Cell Mol Physiol. 2014;307(6):L471-L481.

35. Cheng Z, et al. Mitochondrial translocation of Nur77 mediates cardiomyocyte apoptosis. Eur Heart J. 2011;32(17):2179-2188.

36. Niu G, et al. Orphan nuclear receptor TR3/ Nur77 improves wound healing by upregulating the expression of integrin $\beta 4$. FASEB $J$. 2015;29(1):131-140.

37. Nowakowski TJ, Pollen AA, Sandoval-Espinosa C, Kriegstein AR. Transformation of the radial glia scaffold demarcates two stages of human cerebral cortex development. Neuron. 2016;91(6):1219-1227.

38. Yu Y, et al. The orphan nuclear receptor Nur77 inhibits low shear stress-induced carotid artery remodeling in mice. Int JMol Med 2015;36(6):1547-1555.

39. Au-Yeung BB, et al. A sharp T-cell antigen receptor signaling threshold for T-cell proliferation. Proc
Natl Acad Sci U S A. 2014;111(35):E3679-E3688.

40. Au WS, Payne VA, O'Rahilly S, Rochford JJ. The NR4A family of orphan nuclear receptors are not required for adipogenesis. Int JObes (Lond). 2008;32(2):388-392.

41. Fumoto T, Yamaguchi T, Hirose F, Osumi T. Orphan nuclear receptor Nur77 accelerates the initial phase of adipocyte differentiation in 3T3 L1 cells by promoting mitotic clonal expansion. J Biochem. 2007;141(2):181-192.

42. Malewicz M, et al. Essential role for DNA-PKmediated phosphorylation of NR4A nuclear orphan receptors in DNA double-strand break repair. Genes Dev. 2011;25(19):2031-2040.

43. Lin B, et al. Conversion of Bcl-2 from protector to killer by interaction with nuclear orphan receptor Nur77/TR3. Cell. 2004;116(4):527-540.

44. Thompson J, Winoto A. During negative selection, Nur77 family proteins translocate to mitochondria where they associate with Bcl-2 and expose its proapoptotic BH3 domain. JExp Med. 2008;205(5):1029-1036.

45. Palumbo-Zerr K, et al. Orphan nuclear receptor NR4A1 regulates transforming growth factor- $\beta$ signaling and fibrosis. Nat Med. 2015;21(2):150-158.

46. Duren RP, Boudreaux SP, Conneely OM. Genome wide mapping of NR4A binding reveals cooperativity with ETS factors to promote epigenetic activation of distal enhancers in acute myeloid leukemia cells. PLoS One. 2016;11(3):e0150450.

47. Pei L, Waki H, Vaitheesvaran B, Wilpitz DC, Kurland IJ, Tontonoz P. NR4A orphan nuclear receptors are transcriptional regulators of hepatic glucose metabolism. Nat Med. 2006;12(9):1048-1055.

48. Chao LC, et al. Insulin resistance and altered systemic glucose metabolism in mice lacking Nur77. Diabetes. 2009;58(12):2788-2796.

49. Tessem JS, et al. Nkx6.1 regulates islet $\beta$-cell proliferation via $\mathrm{Nr} 4 \mathrm{a} 1$ and $\mathrm{Nr} 4 \mathrm{a} 3$ nuclear receptors. Proc Natl Acad Sci U S A. 2014;111(14):5242-5247.

50. Qian SW, et al. BMP4-mediated brown fat-like changes in white adipose tissue alter glucose and energy homeostasis. Proc Natl Acad Sci US A. 2013;110(9):E798-E807.

51. Elsen M, et al. BMP4 and BMP7 induce the white-to-brown transition of primary human adipose stem cells. Am J Physiol, Cell Physiol. 2014;306(5):C431-C440.

52. Petruzzelli M, et al. A switch from white to brown fat increases energy expenditure in cancer-associated cachexia. Cell Metab. 2014;20(3):433-447.

53. Tseng YH, et al. New role of bone morphogenetic protein 7 in brown adipogenesis and energy expenditure. Nature. 2008;454(7207):1000-1004.

54. Sidossis LS, et al. Browning of subcutaneous white adipose tissue in humans after severe adrenergic stress. Cell Metab. 2015;22(2):219-227.

55. Patsouris D, et al. Burn induces browning of the subcutaneous white adipose tissue in mice and humans. Cell Rep. 2015;13(8):1538-1544.

56. Knudsen JG, et al. Role of IL-6 in exercise training- and cold-induced UCP1 expression in subcutaneous white adipose tissue. PLOS ONE. 2014;9(1):e84910.

57. Zhan Y, et al. Cytosporone B is an agonist for nuclear orphan receptor Nur77. Nat Chem Biol. 2008;4(9):548-556.

58. Tang QQ, Otto TC, Lane MD. Mitotic clonal expansion: a synchronous process required 
for adipogenesis. Proc Natl Acad Sci U S A. 2003;100(1):44-49.

59. Tang W, Zeve D, Seo J, Jo AY, Graff JM. Thiazolidinediones regulate adipose lineage dynamics. Cell Metab. 2011;14(1):116-122.

60. Bahrami S, Drabløs F. Gene regulation in the immediate-early response process. Adv Biol Regul. 2016;62:37-49.

61. Loebrich S, Nedivi E. The function of activityregulated genes in the nervous system. Physiol Rev. 2009;89(4):1079-1103.

62. Murphy LO, MacKeigan JP, Blenis J. A network of immediate early gene products propagates subtle differences in mitogen-activated protein kinase signal amplitude and duration. Mol Cell Biol. 2004;24(1):144-153.

63. Yuan G, Adhikary G, McCormick AA, Holcroft JJ, Kumar GK, Prabhakar NR. Role of oxidative stress in intermittent hypoxia-induced immediate early gene activation in rat PC12 cells. JPhysiol (Lond). 2004;557(Pt 3):773-783.

64. Adelman K, et al. Immediate mediators of the inflammatory response are poised for gene activation through RNA polymerase II stalling. Proc Natl Acad Sci U S A. 2009;106(43):18207-18212.

65. Haskill S, et al. Characterization of an immediate-early gene induced in adherent monocytes that encodes I kappa B-like activity. Cell. 1991;65(7):1281-1289.

66. Heilbronn L, Smith SR, Ravussin E. Failure of fat cell proliferation, mitochondrial function and fat oxidation results in ectopic fat storage, insulin resistance and type II diabetes mellitus. Int JObes Relat Metab Disord. 2004;28 Suppl 4:S12-S21.

67. McLaughlin T, et al. Enhanced proportion of small adipose cells in insulin-resistant vs insulin-sensitive obese individuals implicates impaired adipogenesis. Diabetologia. 2007;50(8):1707-1715.

68. Pasarica M, et al. Lower total adipocyte number but no evidence for small adipocyte depletion in patients with type 2 diabetes. Diabetes Care.
2009;32(5):900-902.

69. Soccio RE, et al. Genetic variation determines PPAR $\gamma$ function and anti-diabetic drug response in vivo. Cell. 2015;162(1):33-44.

70. Moitra J, et al. Life without white fat: a transgenic mouse. Genes Dev. 1998;12(20):3168-3181.

71. Arner E, et al. Adipocyte turnover: relevance to human adipose tissue morphology. Diabetes. 2010;59(1):105-109.

72. Kim JY, et al. Obesity-associated improvements in metabolic profile through expansion of adipose tissue. JClin Invest. 2007;117(9):2621-2637.

73. Senol-Cosar O, et al. Tenomodulin promotes human adipocyte differentiation and beneficial visceral adipose tissue expansion. Nat Commun. 2016;7:10686.

74. Steinhauser ML, et al. Multi-isotope imaging mass spectrometry quantifies stem cell division and metabolism. Nature. 2012;481(7382):516-519.

75. Rodbell M. Metabolism of isolated fat cells.i. effects of hormones on glucose metabolism and lipolysis. J Biol Chem. 1964;239:375-380.

76. An N, Kang Y. Using quantitative real-time PCR to determine donor cell engraftment in a competitive murine bone marrow transplantation model. JVis Exp. 2013;(73):e50193.

77. Wang QA, et al. Distinct regulatory mechanisms governing embryonic versus adult adipocyte maturation. Nat Cell Biol. 2015;17(9):1099-1111.

78. Huang da W, Sherman BT, Lempicki RA. Systematic and integrative analysis of large gene lists using DAVID bioinformatics resources. Nat Protoc. 2009;4(1):44-57.

79. Chapuy B, et al. Discovery and characterization of super-enhancer-associated dependencies in diffuse large B cell lymphoma. Cancer Cell. 2013;24(6):777-790.

80. Neph S, et al. BEDOPS: high-performance genomic feature operations. Bioinformatics. 2012;28(14):1919-1920.

81. Langmead B, Salzberg SL. Fast gappedread alignment with Bowtie 2. Nat Methods.
2012;9(4):357-359.

82. Varshney A, et al. Genetic regulatory signatures underlying islet gene expression and type 2 diabetes. Proc NatlAcad Sci U S A. 2017;114(9):2301-2306.

83. Grant CE, Bailey TL, Noble WS. FIMO: scanning for occurrences of a given motif. Bioinformatics. 2011;27(7):1017-1018.

84. Mathelier A, et al. JASPAR 2014: an extensively expanded and updated open-access database of transcription factor binding profiles. Nucleic Acids Res. 2014;42(Database issue):D142-D147.

85. Matys V, et al. TRANSFAC and its module TRANSCompel: transcriptional gene regulation in eukaryotes. Nucleic Acids Res. 2006; 34(Database issue):D108-D110.

86. Cline MS, et al. Integration of biological networks and gene expression data using Cytoscape. Nat Protoc. 2007;2(10):2366-2382.

87. DeLuca DS, et al. RNA-SeQC: RNA-seq metrics for quality control and process optimization. Bioinformatics. 2012;28(11):1530-1532.

88. Liao Y, Smyth GK, Shi W. featureCounts: an efficient general purpose program for assigning sequence reads to genomic features. Bioinformatics. 2014;30(7):923-930.

89. Anders S, Huber W. Differential expression analysis for sequence count data. Genome Biol. 2010;11(10):R106.

90. Subramanian A, et al. Gene set enrichment analysis: a knowledge-based approach for interpreting genome-wide expression profiles. Proc Natl Acad Sci U S A. 2005;102(43):15545-15550.

91. Martinez-Santibañez G, Cho KW, Lumeng CN. Imaging white adipose tissue with confocal microscopy. Meth Enzymol. 2014;537:17-30.

92. MacDougald OA. Methods in Enzymology. Methods of adipose tissue biology, part B. Preface. Meth Enzymol. 2014;538:xv.

93. Bogazzi F, et al. Growth hormone is necessary for the p53-mediated, obesity-induced insulin resistance in male C57BL/6J x CBA mice. Endocrinology. 2013;154(11):4226-4236. 